RAIRO Operations Research

RAIRO Oper. Res. 39 (2005) 185-224

DOI: $10.1051 /$ ro: 2006003

\title{
FLOTS ENTIERS ET MULTIFLOTS FRACTIONNAIRES COUPLÉS PAR UNE CONTRAINTE DE CAPACITÉ
}

\author{
Alain Quilliot $^{1}$, Fatiha Bendali $^{1}$ et $^{\text {Eean }}$ Mailfert $^{1}$
}

\begin{abstract}
We present here a Flow/Multicommodity Flow model for Transportation and Production Planning problems. We deal with this model through Lagrangean Relaxation and Hierarchical Decomposition techniques, which involve the resolution of a specific flow with least integral cost sub-problem, and which require the design of some agregation process. We deduce from this analysis several heuristic schemes, and we conclude by discussing numerical experiments.
\end{abstract}

Résumé. Nous modélisons ici plusieurs problèmes de Transport et de Gestion de Flux à l'aide d'un flot entier et d'un multiflot fractionnaire couplés par une contrainte de capacité. Pour le problème ainsi obtenu, nous proposons différents schémas de résolution par relaxation et décomposition, qui induisent la recherche d'un flot auxiliaire dont la partie entière supérieure doit minimiser un certain coût, et qui requièrent la mise en œuvre d'un processus d'agrégation. Nous en déduisons diverses heuristiques que nous testons.

Mots Clés. Flots, Multiflots, Circuits Négatifs, Routage, Transport.

Classification Mathématique. 90C25.

\section{INTRODUCTION}

Les problèmes de synthèse de réseaux issus de la modélisation de systèmes de Transport (voir [13, 22, 24, 33, 51, 53]), de Production (voir [2, 28, 48, 60, 64]), et surtout de Télécommunications $([10,16,17,32,36,63])$, constituent un secteur de recherche en expansion. Des ordinateurs de plus en plus puissants et des systèmes d'informations de plus en plus complets rendent en effet possible à la fois l'acquisition de données complexes (coûts, demandes, ...), et la mise en oeuvre de traitements algorithmiques adaptés à des objets de très grandes tailles. Les grands

Reçu en 2002. Accepté le 12 septembre 2005.

1 Bât. ISIMA, BP 125, Campus des Cézeaux, Université Blaise Pascal, 63173 Aubiere, France; alain.quilliot@isima.fr

(c) EDP Sciences 2006 
mouvements de déréglementation liés aux Transports et aux Télécommunications poussent par ailleurs les opérateurs à repenser, parfois profondément, l'organisation de leurs systèmes.

Configurer un tel système revient, de façon schématique, à déterminer des supports pour acheminer des ressources entre différents couples origine/destination. Ces supports sont, suivant les contextes :

- des vecteurs d'acheminement de l'information ou du signal, des nœuds de commutation;

- des véhicules (bus, navettes, trains, avions) et des plate-formes d'échange;

- des chariots filoguidés, des navettes ou jeux de palettes se déplaçant sur un site industriel ;

- des canalisations, pipelines, ...

La modélisation de ces situations fait souvent apparaître trois grandes classes de problèmes :

- les problèmes de Localisation : [7, 18, 20,30,31]. Il s'agit de localiser les infrastructures d'un système à l'intérieur d'un espace donné. Les méthodes associées sont le plus souvent de nature heuristique. Elles peuvent aussi dériver (méthodes polyédrales) d'une linéarisation du problème et du rôle des modèles linéaires en tant que formalisme universel au sein de la classe NP-Temps ;

- les problèmes de Routage : [3, 5, 19, 61]. Il s'agit de déterminer les cheminements que suivront les différents utilisateurs (signaux, usagers, produits...) du système à configurer, de façon à minimiser des taux de congestion. Ces problèmes, liés à des décisions tactiques ou opérationnelles, souvent issus du contexte des Télécommunications, sont en général traités par des techniques de flots et multiflots (voir $[3,5]$ : méthodes proximales, méthodes de déviation de flots...);

- les problèmes de Dimensionnement et Topologie : [3,11,14,23,25,55,57]. Il s'agit de déterminer les types d'infrastructures qui vont être utilisés par le système, leurs dimensions et leurs positionnements relatifs. L'objectif est alors de minimiser les coûts d'infrastructure, tout en assurant un routage efficace et en satisfaisant d'éventuelles contraintes de fiabilité (survivability : $[6,10])$. Ces problèmes, liés à des niveaux de décision stratégique, sont très complexes, tant d'un point de vue pratique que théorique [45]. Ils peuvent faire l'objet de traitements heuristiques ou induire la mise en œuvre de techniques polyédrales ou de modèles de multiflots.

Dans tous les cas, le caractère prospectif des problèmes posés, le niveau d'approximation existant sur les entrées des modèles et la nature forcément réductrice de ceux-ci, font qu'il est de fait moins important d'obtenir des solutions numériques exactes, que de savoir gérer les principaux points durs de ces modèles de façon robuste.

La classe des problèmes que nous étudions ici s'inscrit dans cette dernière catégorie des problèmes de dimensionnement et de topologie et correspond à la formulation suivante : 


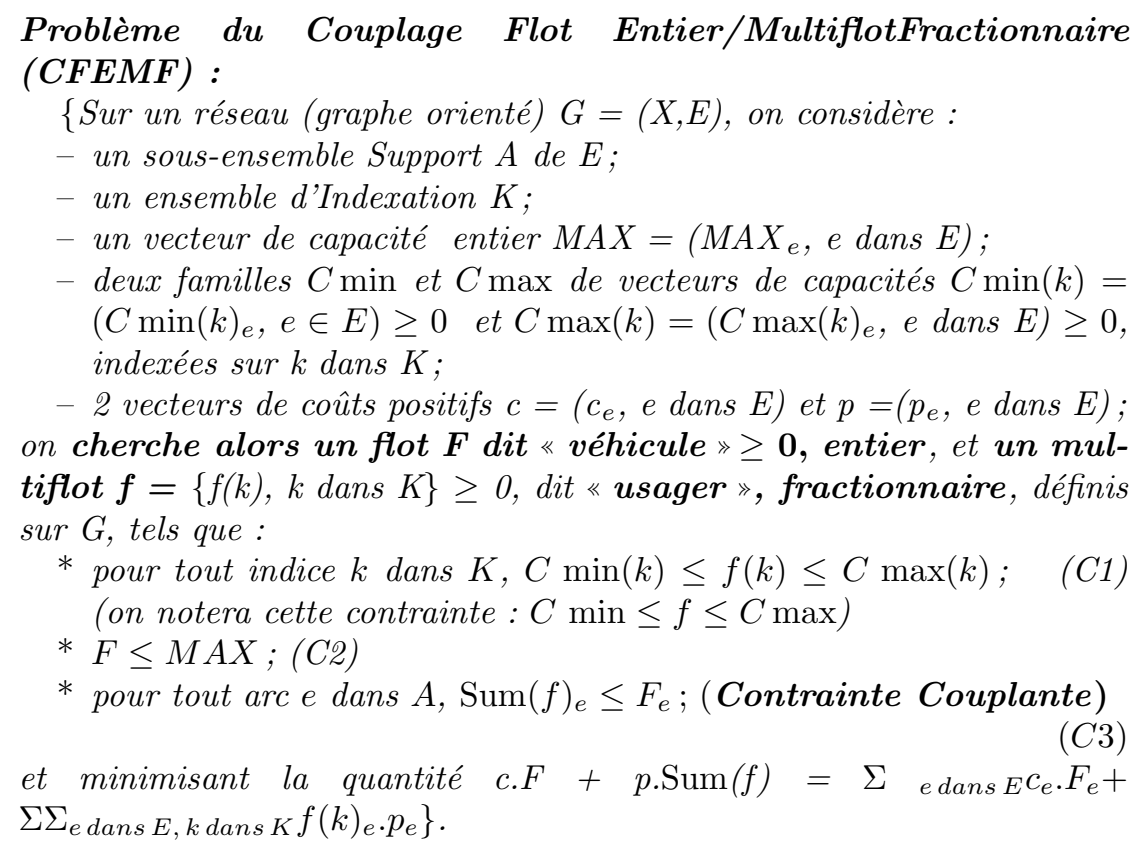

Un cas très important est celui où l'ensemble d'indexation $K$ correspond de fait à une famille de couples de sommets origines et destinations de $X$, et où, pour chaque couple $k=(o, d)$ dans $K$, la contrainte $(\mathrm{C} 1)$ se réécrit :

le flot $f(k)$ achemine une quantité de flot $D_{k}$ depuis o vers $d$;

on note alors CFEMF-OD le problème ainsi obtenu.

Remarque. La quantité $\operatorname{Sum}(f)$ est définie de façon algébrique. Mais le multiflot $f$ étant assujetti à être positif, la contrainte $(\mathrm{C} 3)$ peut néanmoins être vue comme une contrainte de capacité classique.

Formellement, ce problème linéaire en nombres mixtes est proche des modèles de type CFA (Capacitated Flow Assignment) : $[9,14,25,55]$. Ceux-ci, hérités du contexte des Télécommunications, portent sur un vecteur entier $F$ indexé sur les arcs du réseau $G$, et sur un multiflot $f$ qui achemine des demandes émanant d'un ensemble $K$ de couples de sommets origines/destinations, $F$ dominant $f$ sur l'ensemble des arcs de $G$ et satisfaisant d'éventuelles contraintes de connectivité. La quantité à minimiser est alors l'expression d'un coût d'infrastructure linéaire et d'un coût (mesure de congestion) de qualité de service, éventuellement non linéaire.

Il comporte néanmoins des spécificités, justifiant un mode de traitement propre. En effet, comme cela sera illustré dans la section suivante, ce modèle dérive plus de considérations relatives aux systèmes de transport et de production que du contexte des Télécommunications. La contrainte de flot sur $F$ exprime que celuici représente non pas un systèmes de connections fixes, mais un objet (ensemble de véhicules) circulant, que les usagers représentés par le multiflot $f$ peuvent se 
passer d'utiliser. Il s'ensuit que la contrainte de couplage (C3) ne concerne que certains arcs (les arcs de l'ensemble Support $A$ ) du réseau. Il en découle :

- qu'il devient difficile d'introduire des coupes sur $F$, traduisant le fait que les arcs supports de $F$ doivent permettre de connecter tout couple de sommets $(o, d)$ de la famille $K$, et d'en déduire une approche polyédrale telle que pratiquée en $[9,14,26,52,62]$;

- que la contrainte d'intégrité sur $F$ traduit le besoin (très apparent dans les applications liées au Transport) qu'ont les utilisateurs de se regrouper afin de se partager un réseau d'infrastructure faiblement maillé par rapport au réseau initial $G=(X, E)$. La relaxation de la contrainte d'intégrité sur $F$ fournit dès lors ici, dans la plupart des cas, de très mauvais résultats, reflétant cette absence de partage, et ce contrairement à ce qui est observable dans le cas des problèmes liés aux Télécommunications (voir par exemple [25];

- que le flot agrégé $\operatorname{Sum}(f)$, synthèse de la mutualisation par les usagers de leurs stratégies d'acheminement, devient dès lors l'Objet Maître à l'intérieur du problème CFEMF. Il est dès lors souhaitable, dans la perspective d'un traitement heuristique de ce problème, de pouvoir agir directement sur $\operatorname{Sum}(f)$ et $F$, et ce en tirant parti des spécificités des espaces de flots.

Ces constatations sont le moteur de notre démarche. Ce que nous voulons ici est concevoir un procédé algorithmique de nature heuristique, propre à traiter des instances de CFEM de tailles importantes, agissant directement sur le flot agrégé $\operatorname{Sum}(f)$ à l'aide de procédures de transformation locale à base de cycles et de chemins. Dans un premier temps (Sect. 2) nous décrivons 3 cas dont la modélisation renvoie à CFEMF, et qui permettent d'appréhender le sens de l'ensemble support A et de la contrainte de flot imposée à $F$. Nous présenterons ensuite (Sect. 3), 3 approches très générales pour traiter ce problème CFEMF :

- par relaxation lagrangienne de la contrainte de flot en $F$;

- par relaxation lagrangienne de la contrainte de couplage (C3);

- par décomposition hiérarchique, l'objet multiflot $f$ étant considéré comme l'objet Maitre ;

qui toutes recentrent le problème autour de l'objet agrégé $\operatorname{Sum}(f)$. Ce recentrage questionne la gestion de l'articulation entre $f$ et $\operatorname{Sum}(f)$ (processus d'agrégation/ désagrégation), et celle d'un flot fractionnaire compatible avec des capacités et dont la partie entière supérieure minimise un certain coût. Nous proposons une heuristique pour ce dernier problème (Sect. 4.1) puis présentons (Sect. 4.2) un traitement du problème CFEMF-OD par action directe sur le flot agrégé $\operatorname{Sum}(f)$. Dans les deux cas, les algorithmes ainsi présentés tirent parti, tant pour la gestion de $F$ que de $\operatorname{Sum}(f)$ et $f$, des procédés classiques de transformation de flots par chemins et cycles. Nous concluons cet article par la présentation d'un ensemble d'expérimentations numériques (Sect. 5). 


\section{Modèles et interprétAtions}

\subsection{Principales nOtations}

Un réseau $G$, d'ensemble de sommets $X$ et d'ensemble d'arcs $E$ est noté $G=$ $(X, E)$. La matrice d'incidence arcs/sommets de ce réseau est notée $M(G)$ : si l'arc $e=[x, y]$ va de $x$ vers $y$, alors $M(G)_{x, e}=1$ et $M(G)_{y, e}=-1$.

Un multiflot $f$, défini sur $G$ et dont les flots composants sont indexés sur un ensemble $K$, est noté $f=\{f(k), k$ dans $K\}$. On note Sum $(f)$ le Flot Agrégé défini $\operatorname{par} \operatorname{Sum}(f)=\Sigma_{k \text { dans } K} f(k)$.

Si $\gamma$ est un cycle (chemin), orienté de $G$, nous notons $\phi_{\gamma}$ le flot-cycle (flotchemin) associé à $\gamma$, c'est-à-dire le vecteur flot qui vaut 1 sur les arcs de $\gamma$ orientés comme $\gamma,-1$ sur les arcs de $\gamma$ orientés contre $\gamma$, et 0 ailleurs.

On dit qu'un flot $f$ achemine une quantité $\mathrm{D}$ de $o$ vers $d$ si $f$ s'écrit $f=D . \phi_{\gamma}$, où $\gamma$ est un chemin allant de $o$ vers $d$.

Si $x$ et $y$ sont 2 sommets de $G$, et si $D$ est un nombre réel, nous notons $D^{x, y}$ le vecteur indexé sur $X$, égal à $D$ pour l'indice $x$, à $-D$ pour l'indice $y$, et à 0 ailleurs. Si $z$ est un vecteur indexé sur $X$ et si $A$ est un sous-ensemble de $X$, alors $z_{A}$ est la trace de $z$ sur $A$. On note $z^{+}$la partie positive du vecteur $z$, et $z^{-}$sa partie négative.

\subsection{Modélisation D’un PROBlème DE TOURnÉEs À L'Aide D'Un RÉSEAU DYNAMIQUE}

Sur un graphe orienté $H=(Z, U)$ représentatif d'un réseau urbain, nous considérons des sommets particuliers $y_{1}, y_{2}, \ldots, y_{m}$ correspondant à l'emplacement de sites de production (lieux de travail). À chaque sommet $x \in Z$, et à chaque site $y_{k}$ est associée une quantité fixe $d_{x, y k, t j}$ d'usagers à acheminer depuis $x$ vers $y_{k}$, avant la date $t_{j}, j \in J^{k}$. La durée d'acheminement ne doit pas excéder un seuil $T_{x, y k}$. On souhaite organiser un système de navettes, qui, pour un coût minimal, va assurer ces acheminements. On suppose que :

- chaque tournée de véhicule débute et s'achève en un sommet Dépôt unique;

- à chaque véhicule correspond une capacité unique, et l'unité de mesure choisie pour les flux d'usagers est telle que cette capacité est égale à 1, et que les quantités $d_{x, y k, t j}$ sont fractionnaires;

- le coût du système dépend linéairement du nombre de véhicules impliqués et des durées des tournées;

- les déplacements des usagers se font par combinaison de déplacements en véhicule et de déplacements à pied. À chaque arc $e$ de $\mathrm{H}$ sont donc associées une durée $l_{p}(e)$, à pied, et une durée $l_{v}(e)$, en véhicule.

Un réseau dynamique associé

Le problème se modélise sous forme CFEMF-OD grâce à un réseau dynamique (voir [4] pour la notion de réseau dynamique) qui intègre les contraintes temporelles sur les tournées. On considère (Discrétisation du Temps) une unité de 
temps discrète $\delta$ et un entier $N$, tels que la période correspondant à l'ensemble des acheminements se situe entre les instants 0 et $N \delta$. Pour chaque arc $e$ de $H$, on pose :

$$
l_{p}^{*}(e)=\left\lceil l_{p}(e) / \delta\right\rceil ; l_{v}^{*}(e)=\left\lceil l_{v}(e) / \delta\right\rceil ; t_{j}^{*}=\left[t_{j} / \delta\right]
$$

À un sommet quelconque $x$ de $Z$, on associe $(N+1)$ copies de $x$, indexées de 0 à $N$, qui représentent l'état de $x$ aux instants $0, \delta, \ldots, N \delta$. On rajoute un sommet $D$ (dépôt), destiné à l'écriture des lois de Kirschoff, et on pose : $X=\left\{x_{r}, x \in X\right.$, $r \in 0, \ldots, N\} \cup\{D\}$. Sur l'ensemble de sommets $X$, on définit :

- des arcs $\left[x_{r}, x_{r+1}\right]$ pour $x \in Z$ et $r \in 0, \ldots, N-1$, dédoublés selon un étiquetage navette, et $\grave{a}$-pied;

- des arcs étiquetés navette $\left[D, \operatorname{Depot}_{r}\right]$ et $\left[\operatorname{Depot}_{r}, D\right], r \in 0, \ldots, N$;

- des arcs "navette" $\left[x_{r}, z_{r+l^{*} v(e)}\right]$, pour $e=[x, z] \in U$ et pour $r=1 \ldots m$ tels que $0 \leq r \leq N-l_{v}^{*}(e)$;

- des arcs $\left[x_{r}, z_{r+l^{*} p(e)}\right]$ étiquetés à-pied pour $e=[x, z] \in U$ et $r$ tels que $0 \leq r \leq N-l_{p}^{*}(e)$;

L'ensemble des arcs étiquetés navette est noté $A$.

À chaque arc $e$ du réseau dynamique $G=(X, E)$ ainsi défini, on associe un coût $L_{e}$ en posant :

- $L_{e}=l_{v}(e)$ si $e$ est de la forme $\left[x_{r}, z_{r+l^{*} v(e)}\right]$;

- $L_{e}=$ une constante $\mu$ si $e$ est de la forme $\left[x_{r}, x_{r+1}\right]$ : ce coût d'attente, est un coût de fonctionnement;

- $L_{e}=$ une constante $\alpha$ si $e$ est du type $\left[D\right.$, Depot $\left._{r}\right]$ : ce coût est lié au nombre de véhicules utilisés;

$-L_{e}=0$ ailleurs.

Le modèle induit

Sur $G=(X, E)$ ainsi construit, on cherche alors un flot $F \geq 0$ entier, représentant les parcours de navettes, et un multiflot $f=\{f(k, j), \bar{k}=1 \ldots m, j$ dans $\left.J^{k}\right\} \geq 0$, représentant les parcours des usagers, tels que :

- $F$ est nul sur les arcs à-pied;

- $f(k, j)$ achemine la demande $d_{x, y k, t j}$ du sommet $x_{w *(x, k, j)}$, où $w *(x, k, j)=$ $\left\lceil\left(t_{j}-T_{x, y k}\right) / \delta\right\rceil$, vers le sommet $y_{t * j}$;

- $\operatorname{Sum}(f)_{e}=\Sigma_{k, j} f(k, j)_{e} \leq F_{e}$, pour chaque arc $e$ de $E$ étiqueté navette et qui minimisent une quantité $L . F=\Sigma_{(e \text { dans } A)} L_{e} . F_{e}$.

Ce modèle, de type CFEMF-OD, permet l'expression implicite des contraintes temporelles et des contraintes de synchronisation, qui dérivent de la possibilité qu'a un usager d'avoir recours à plusieurs véhicules. Il serait possible de l'enrichir en intégrant un coût $p . f$ de Qualité de Service relatif aux usagers, qui exprimerait l'intérêt qu'ont ceux-ci à se voir proposer les parcours les plus rapides possibles.

On vérifie que la reconstitution d'un système cohérent de tournées découle directement de la connaissance du flot $F$. L'approximation induite par les remplacements respectifs de chaque temps $l_{v}(e)$ et $l_{p}(e)$ par $\delta . l_{v}^{*}(e)$ et $\delta . l_{p}^{*}(e)$ peut toutefois produire, dans le cas où $\delta$ est assez grand, des parcours relativement lents. Un 
traitement de ce problème des Navettes s'appuyant sur la modélisation ci-dessus, s'effectuera dès lors en 2 étapes :

$1^{\text {re }}$ étape : résolution de l'instance de CFEM-OD associée;

$2^{\mathrm{e}}$ étape : décomposition de $F$ en un système de tournées;

optimisation des temps de passage des véhicules durant ces tournées (graphicage).

On voit ici le rôle du vecteur $F$, qui représente la circulation de la flotte de véhicules, et celui de l'ensemble $A$, qui identifie ceux des déplacements des usagers à l'intérieur du réseau qui sont effectués à l'aide de ces véhicules. Relâcher la contrainte d'intégrité sur $F$ revient ici à offrir à chaque usager sa propre fraction de véhicule, et induit donc une dislocation des parcours individuels, soit le contraire de ce qui est recherché.

\subsection{Modélisation D’un système de Production}

On considère ici un atelier dont il faut planifier l'activité cyclique sur $n+1$ périodes séparées par des interpériodes, pour produire des biens $b_{1}, b_{2}, \ldots, b_{m}$. Ces biens font partie d'une même gamme de produits, ce qui signifie qu'ils sont structurellement proches les uns des autres, et qui permet de les considérer de façon unifiée dès lors qu'il est question de capacités de production ou de coûts de stockage. On suppose que :

- la demande en biens $b_{k}$ à la fin de la période $i$ est $d_{i, k}$;

- le coût unique de stockage d'une unité de produit est $\alpha$;

- la capacité de stockage sur l'ensemble des produits pour une interpériode est $\beta$;

- la capacité totale de production de l'atelier est $c$. Elle peut être augmentée à un niveau $d$ moyennant la location d'une machine spécifique au coût $\gamma$ par période. Les opérations de montage et démontage de cette machine doivent être effectuées lors des interpériodes aux coûts respectifs de $\gamma_{1}$ et $\gamma_{2}$.

L'activité de l'atelier (gestion des stocks, de la production avec ou sans machine supplémentaire...) doit dès lors être planifiée de façon à minimiser les coûts tout en satisfaisant les demandes.

On utilise, afin de définir un modèle CFEMF de ce problème, le réseau dynamique construit comme suit :

- pour chaque période $i=0, \ldots, n$, on crée deux sommets $x_{i}, y_{i}$ (début et fin de période), auxquels on ajoute trois sommets machine, client, production;

- sur l'ensemble $X$ de sommets ainsi construit, on crée :

- pour chaque $i=0, \ldots, n$, les $\operatorname{arcs}\left[y_{i}\right.$, client $]$, [production, $\left.x_{i}\right]$ et $\left[x_{i}, y_{i}\right]$, ce dernier arc étant dédoublé selon 2 étiquettes Sans-Machine et Avec-Machine : ces arcs vont respectivement porter les quantités de biens livrées, demandées et produites aux différentes périodes; 
- pour chaque $i=0, \ldots, n$, un arc $\left[y_{i}, x_{i+1}\right]$ (à cause de la cyclicité, l'addition est prise modulo $n+1$ ), dédoublé selon 2 étiquettes SansMachine et Avec-Machine : ces arcs vont respectivement porter les quantités de biens stockées et l'état de la machine entre les périodes $i$ et $i+1$;

- pour chaque $i=0, \ldots, n$, un arc $\left[\right.$ machine, $\left.x_{i}\right]$ et un arc $\left(y_{i}\right.$, machine $]$, qui porteront l'information relative au montage et au démontage de la machine en amont et en aval de la période $i$;

- un arc [client, production] de retour.

Sur ce réseau $G=(X, E)$, on cherche alors un multiflot $f=\{f(k), k=1 \ldots m\} \geq 0$, représentatif de la circulation des biens $b_{1}, b_{2}, \ldots, b_{m}$, et un flot $F$ en $\{0,1\}$, représentatif de l'état de la machine, tels que :

- $F=0$ sur tout arc dont une extrémité est client ou production, ainsi que sur les arcs Sans-Machine;

- $f=0$ sur tout arc dont une extrémité est machine, ainsi que sur les arcs Avec-Machine du type $\left[y_{i}, x_{i+1}\right]$;

- $f(k)$ est égal à $d_{i, k}$ pour tout arc $\left[y_{i}\right.$, Client $]$;

- pour tout arc $e$ de la forme $\left[y_{i}, x_{i+1}\right]$ et étiqueté Sans-Machine, $\operatorname{Sum}(f)_{e} \leq$ $\beta ;$ (contrainte de Stockage)

- pour tout arc $e=\left(x_{i}, y_{i}\right)$ et étiqueté Sans-machine, $\operatorname{Sum}(f)_{e} \leq c_{e} ;\left(1^{r e}\right.$ contrainte de capacité)

- pour tout arc (ensemble Support) $e=\left(x_{i}, y_{i}\right)$, étiqueté Avec-machine, $\operatorname{Sum}(\mathrm{f})_{e} \leq F_{e} \cdot\left(d_{e}-c_{e}\right)$

- et minimisant la quantité : $\gamma_{1} \cdot \Sigma_{i} F_{[\text {mach }, y i]}+\gamma_{2} . \Sigma_{i} F_{[x i, \text { mach }]}+\gamma \cdot \Sigma_{i} F_{[x i, y i]}+$ $\left.\Sigma_{i, k} \alpha \cdot f(k)_{[y i, x i+1]}\right)$.

Remarque. La contrainte de stockage et la $1^{\text {re }}$ contrainte de capacité contraignent entre elles les composantes du multiflot $f$, ce qui déborde du cadre proposé pour CFEMF. Les méthodes proposées en sections 3 et 4 demeureront cependant adaptables, et ce d'autant plus aisément que ces contraintes portent de fait sur l'objet agrégé $\operatorname{Sum}(f)$, qui sera l'objet principal à l'intérieur de ces méthodes.

\subsection{Modélisation DU PROBlème DU CIRCUit HAMiltonien SOUS LA FORME CFEMF}

Soit donc $H=(Y, U)$ un réseau, supposé fortement connexe, et soit $x_{0}$ un sommet de référence de $H$. Construisons un réseau auxiliaire $G=(X, E)$ comme suit :

- $X=Y \cup Y^{\prime}$ où $Y^{\prime}$ est une copie de $Y$;

- $E=\left\{\left[x, x^{\prime}\right], x \in Y, x^{\prime}\right.$ étant sa copie dans $\left.Y^{\prime}\right\} \cup\left\{\left[x^{\prime}, y\right]\right.$, pour tous les $\operatorname{arcs}[x, y] \in U\} \cup\left\{\left[x_{o}, x^{\prime}\right], x^{\prime} \in Y^{\prime}\right\}$.

On définit l'ensemble support $A$ comme formé des arcs de la forme $\left[x^{\prime}, y\right], x^{\prime} \in$ $Y^{\prime}, y \in Y$, et on pose $n=[X]$. Chercher un circuit hamiltonien dans $H$ 
signifie alors chercher sur $G$ un flot entier $F \geq 0$, et un flot fractionnaire $f \geq 0$ tels que :

- $\forall x^{\prime}$ dans $Y^{\prime}, f_{\left[x o, x^{\prime}\right]}=1 / n$;

- pour tout arc $e$ dans $A, \operatorname{Sum}(f)_{e} \leq F_{e}$;

- $F_{e}=1$ pour tout arc $e$ de la forme $e=\left[x, x^{\prime}\right], x \in Y$.

On en déduit, dans la continuité des résultats relatifs à la complexité de la synthèse de réseau (voir [45]) :

Proposition 1. Le problème CFEMF, considéré dans le cas particulier où $k=1$ est NP-difficile.

\section{SChÉMAs GÉNÉRAuX DE RÉSOLUTION POUR CFEMF}

Soit donc, conformément aux notations de la section 1 , un réseau $G=(X, E)$, pour lequel sont donnés :

- un sous-ensemble Support $A$ de $E$;

- un ensemble d'Indexation $K$,

- un vecteur Capacité entier $M A X=\left(M A X_{e}, e\right.$ dans $\left.E\right)$,

- deux familles $C \min$ et $C \max$ de vecteurs Capacité $C \min (k)=$ $\left(C \min (k)_{e}, e\right.$ dans $\left.E\right) \geq 0$ et $C \max (k)=\left(C \max (k)_{e}, e\right.$ dans $\left.E\right) \geq 0$, indexées sur $k$ dans $K$,

- 2 vecteurs Coût positifs $c=\left(c_{e}, e\right.$ dans $\left.E\right)$ et $p=\left(c_{e}, e\right.$ dans $\left.E\right)$.

On cherche alors, sur $G$ (problème CFEMF) :

un flot $F \geq 0$, entier, et un multiflot $f=\{f(k), k$ dans $K\} \geq 0$, respectivement compatibles avec le vecteur $M A X$ et avec les familles $C \min$ et $C \max$, tels que :

pour tout arc e dans $A, \operatorname{Sum}(f)_{e}=\Sigma_{k \text { dans } K} f(k)_{e} \leq F_{e}$ (Contrainte couplante C3) et tels que la quantité c.z $+p \cdot \operatorname{Sum}(f)$ soit la plus petite possible.

Notations additionnelles.

On note $V$ la valeur optimale du problème CFEMF et $V^{*}$ la valeur optimale du problème $\mathbf{C F E M F}^{*}$ déduit de $\mathbf{C F E M F}$ par relaxation de la contrainte d'intégrité sur $F$.

Un flot entier $F^{*}$ étant donné, on note $\mathbf{C F E M F}{ }^{F *}$ le problème $\mathbf{C F E M F}$ restreint en imposant $F=F^{*}, V^{F *}$ la valeur optimale associée, et $\boldsymbol{D} \boldsymbol{U}^{F *}$ le dual de CFEMF $^{F *}$.

Un multiflot $f^{*}$ étant donné, on note $\mathbf{C F E M F}_{f *}$ le problème $\mathbf{C F E M F}$ restreint en imposant $f=f^{*}, V_{f *}$ la valeur optimale associée et $\boldsymbol{D} \boldsymbol{U}_{f *}$ le dual de CFEMF $_{f *}$.

On note $E^{\infty}$ l'ensemble des arcs $e$ de $E$ tels que $M A X_{e}=+\infty$.

Cette section traite donc de l'application au problème CFEMF de schémas de décomposition et de relaxation, et cela afin d'induire des propositions d'heuristiques. Comme il a été dit, relâcher la contrainte d'intégrité sur $F$ déforme fortement le problème, et il est difficile de générer des coupes sur $F$ qui compensent cette relaxation. Du fait du rôle central joué par le vecteur agrégé $\operatorname{Sum}(f)$, qui 
synthétise le partage de la ressource $F$ par les flots usagers $f(k), k \in K$, on va étudier des modes de décomposition qui considèrent ce vecteur $\operatorname{Sum}(f)$ comme objet principal du problème, à savoir :

- un procédé de décomposition Maître/Esclave DME (Benders, Sect. 3.1), qui considère $f$ comme l'objet Maître et exploite les propriétés des flots entiers. Ce procédé va faire apparaître un problème auxiliaire $\mathbf{P}$-aux de "Multiflot de Coût Entier Minimal", qui sera approfondi en section 4.1;

- un procédé de relaxation lagrangienne DRCOUP de la contrainte couplante (C3), (Sect. 3.2), et un procédé de relaxation lagrangienne DRFLOT de la contrainte de flot sur $F$, (Sect. 3.3), qui induiront des résultats similaires et l'appel au traitement d'une instance du problème auxiliaire P-aux.

L'analyse à laquelle nous nous livrerons tendra à suggérer que les comportements de ces trois procédés sont assez voisins et qualitativement satisfaisants, et que les cas pour lesquels ils débouchent sur une valeur optimale sont les mêmes (Ths. 1 et 2). Ceci justifiera (Sects. 4.1 et 4.2) que nous cherchions à nous appuyer sur ces procédés, et notamment sur le mode de décomposition Maître/Esclave DME, qui présente l'avantage d'opérer sur des solutions réalisables de CFEMF, de façon à en extraire une heuristique opérationnelle.

\subsection{SChÉma DME : DÉCOMPOSITION MAÎTRE-ESClAVE}

Soit donc $(F, f)$ une solution réalisable de CFEMF, choisie telle que $F$ soit solution optimale du problème restreint $\mathbf{C F E M F}_{f}$. Correspond alors à ce couple $(F, f)$ une solution optimale $\left(\mu=\left(\mu_{x}, x \in X\right), \alpha=\left(\alpha_{e}, e \in E\right) \geq 0, \lambda=\left(\lambda_{e}\right.\right.$, $e \in A) \geq 0)$ du dual $\mathbf{D U}_{f}$ de $\mathbf{C F E M F}_{f}$. Améliorer le couple $(F, f)$ signifie modifier $f$ de façon à le maintenir positif, compatible avec les familles $C$ min et $C \max$, et à diminuer la quantité :

$$
\lambda .\lceil\operatorname{Sum}(f)\rceil+p \cdot \operatorname{Sum}(f)-\alpha \cdot M A X .
$$

La réciproque est bien entendu fausse. Cette remarque nous conduit à introduire un problème auxiliaire :

Problème $\boldsymbol{P}-\boldsymbol{a u x}(\lambda):\{$ Le vecteur $\lambda, \geq 0$ étant donné, indexé sur $E$ et nul sur $E$ - $A$, chercher un multiflot $f \geq 0$, compatible avec $C \min$ et $C \max$ et minimisant la quantité : $\lambda .\lceil\operatorname{Sum}(f)\rceil+p . \operatorname{Sum}(f)\}$

et à proposer le schéma algorithmique suivant :

Schéma algorithmique DME (Décomposition Mâ̂tre/Esclave)

Initialiser $f$, réalisable pour le problème CFEMF ; Not Stop ; $\Delta:=+\propto$; Tant que $\neg$ Stop faire

Résoudre CFEMF $_{f}$ et extraire la composante primale $F$ et la composante duale $\lambda$ associées ;

$\Delta:=\mathrm{c} . \mathrm{F}+p . \operatorname{Sum}(f) ;$ Mettre à jour Stop (en cas de dégradation ou de surplace prolongé de $\Delta$ ); 
Perturber $f$ de façon à diminuer la quantité $\lambda$. $\lceil\operatorname{Sum}(f)\rceil+p . \operatorname{Sum}(f)$ tout en maintenant $f$ compatible avec $C \min$ et $C \max$; (I1)

Si l' instruction (I1) échoue alors Stop;

Surplace Prolongé signifie ici que l'on n'enregistre plus d'amélioration de la quantité $c . F+p . \operatorname{Sum}(f)$, supérieure à un seuil $\varepsilon$, depuis $N$ d'itérations de la boucle principale : $N$ et $\varepsilon$ sont alors des paramètres du processus.

Nous reviendrons sur le problème $\mathbf{P}$-Aux en sections 4 et 5 .

Remarque. Ce schéma DME est heuristique, puisque l'évolution de $f$ au cours d'une itération est susceptible d'aller en sens contraire des perturbations appliquées au cours des itérations précédentes. Il serait bien sûr possible de réécrire $\mathbf{D M E}$ sous forme d'un processus de génération incrémentale de coupes de la forme :

$$
\lambda .\lceil\operatorname{Sum}(f)\rceil+p \cdot \operatorname{Sum}(f) \geq S, \quad(\alpha)
$$

qui serait alors exact (modulo les problèmes liés à la vitesse de convergence du processus). Mettre en œuvre ce processus impliquerait toutefois de savoir gérer simultanément un grand nombre de coupes de type $(\alpha)$ ci-dessus.

\subsection{Schéma DRCOUP : Relaxation de la CONTRAinte Couplante}

Relaxer la contrainte couplante (C3) signifie introduire un vecteur de Lagrange $\lambda \geq 0$, indexé sur $E$ et nul sur $E-A$, et poser :

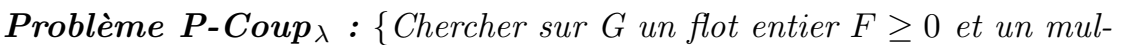
tiflot $f=\{f(k), k$ dans $K\} \geq 0$, compatibles avec leurs capacités, et qui minimisent la quantité c.F $+p . \operatorname{Sum}(f)-\lambda .(F-[\operatorname{Sum}(f)])\}$.

On note $B_{\lambda}$ la valeur optimale de $\mathbf{P}-\operatorname{Coup}_{\lambda}$ et on pose $: B=\operatorname{Sup}_{(0 \leq \lambda)} B_{\lambda}$. Le problème $\mathbf{P}_{-} \mathbf{C o u p}_{\lambda}$ se sépare en une instance du problème $\mathbf{P}$-aux $(\lambda)$, et en un problème de flot entier de coût minimum. On peut se limiter à $\lambda$ tel que $B_{\lambda}>-\infty$, soit tel qu'il n'existe pas de circuit négatif pour les coûts $c-\lambda$ dans le réseau $G^{\infty}=\left(X, E^{\infty}\right)$.

Quand $E^{\infty}=E$, on peut donc associer à $\lambda$ un vecteur potentiel $\Pi$, indexé sur $X$ et tel que : П. $M(G) \leq c-\lambda$. La correspondance qui à $\lambda$ associe $B_{\lambda}$ étant alors croissante en $\lambda$, on obtient :

$$
\begin{array}{r}
B=\operatorname{Sup}_{\Pi \text { telsque } \Pi \cdot M(G) \leq c} \operatorname{Inf}_{f \text { tels } C \min \leq f \leq C \max }(p \cdot \operatorname{Sum}(f)+ \\
\left.\quad(c-\Pi \operatorname{\Pi uM}(G))_{A} \cdot\left\lceil\operatorname{Sum}(f)_{A}\right\rceil\right) .
\end{array}
$$

Dans le cas inverse, ou peut supposer que le vecteur $M A X$ est borné, quitte à introduire des capacités fictives suffisamment grandes, et $B$ se calcule alors selon le schéma suivant :

Initialisation de $\lambda$; Not Stop;

Tant que Not Stop faire

Résoudre la composante en $F$ de $\mathbf{P}-\operatorname{Coup}_{\lambda}$; soit $F_{\lambda}$ la solution obtenue; 
Résoudre la composante en $f$ de $\mathbf{P}-\operatorname{Coup}_{\lambda}$; soit $f_{\lambda}$ la solution; Mettre à jour Stop;

Si Not Stop alors

Calculer une quantité Pas; $\lambda:=\operatorname{Sup}\left(0, \lambda-\right.$ Pas. $\left(F_{\lambda}-\right.$ $\left.\left\lceil\operatorname{Sum}\left(f_{\lambda}\right)\right\rceil\right)_{A}$

Dans tous les cas, il nous est suggéré le schéma de résolution DRCOUP suivant pour le problème CFEMF :

phase 1 : calcul de $B$;

phase 2 : extraction par projection d'une solution de CFEMF.

Soit $\lambda_{o}$ tel que $B_{\lambda o}$ peut être pris comme approximation de $B$; soit $f_{o}$ la solution de la composante en $f$ du problème $\mathbf{P}-\mathbf{C o u p}_{\lambda}$; résoudre le problème $\mathbf{C F E M F}_{f o}$; soit $F_{o}$ la solution obtenue; prendre le couple $\left(F_{o}, f_{o}\right)$ comme solution approchée du problème CFEMF.

\subsection{SchÉma DRFLOT : Relaxation De la CONTRAinte De Flot sur $F$}

Relaxer la contrainte de flot sur $F$ signifie poser, pour tout vecteur de Lagrange $\mu$ indexé sur les sommets de $G$ :

Problème P-Flot $_{\mu}:\{$ Chercher sur $G$ un vecteur entier $F \geq 0$ et un multiflot $f=\{f(k), k \in K\} \geq 0$, compatibles avec leurs capacités, tels que $\operatorname{Sum}(f)_{A} \leq F_{A}$, et minimisant c.F + p.Sum $\left.(f)-\mu . M(G) . F\right\}$.

On note $D_{\mu}$ la valeur optimale de $\mathbf{P}$-Flot ${ }_{\mu}$ et on pose : $D=\operatorname{Sup}_{\mu} D_{\mu}$.

On peut se limiter aux vecteurs $\mu$ assurant la finitude de $D_{\mu}$, soit tels que pour tout arc $e$ dans $E^{\infty}$, on ait $(c-\mu(M G))_{e} \leq 0$. Pour tout vecteur $\mu$, la solution optimale $\left(F_{\mu}, f_{\mu}\right)$ de $\mathbf{P}$-Flot ${ }_{\mu}$ est alors telle que :

pour tout $\operatorname{arc} e$ dans $A$, tel que $0 \leq\left(c-\mu M(G)_{e}\right.$, on a $F_{e}=\left\lceil\operatorname{Sum}(f)_{e}\right\rceil$; pour tout arc $e$ dans $E-A$, tel que $0 \leq(c-\mu M(G))_{e}$, on a $F_{e}=0$; pour tout arc $e$ dans $E$, tel que $(c-\mu M(G))_{e} \leq 0$, on a $F_{e}=M A X_{e}$.

Le problème $\mathbf{P}-$ Flot $_{\mu}$ se réécrit alors :

\{Trouver un multiflot $f \geq 0$, compatible avec $C \min$ et $C \max$, tel que $\operatorname{Sum}(f)_{A} \leq M A X_{A}$, et qui minimise : $p \cdot \operatorname{Sum}(f)-\Sigma_{e \text { dans } A / 0 \leq(c-\mu M(G)) e}$ $\left.(c-\mu M(G))_{e} \cdot\left\lceil\operatorname{Sum}(f)_{e}\right\rceil+\Sigma_{e \text { dans } E /(c-\mu M(G)) e \leq 0}(c-\mu M(G))_{e} \cdot M A X_{e}\right\}$.

On reconnaît là une instance du problème $\mathbf{P}$-aux, enrichie d'une contrainte globale sur le vecteur $\operatorname{Sum}(f)$.

Faisant l'hypothèse que nous savons traiter P-aux, nous pouvons alors proposer, pour traiter CFEMF, un processus DRFLOT similaire à celui de la section 2.2. Ce processus comporte en première phase un calcul (éventuellement approché) de $D$, suivi de l'extraction d'une solution réalisable de CFEMF au moyen d'un algorithme de flot entier de coût minimum. 


\subsection{UN EXEMPlE : LE TSP TRIANGUlaire}

Soit $K_{n}$ le réseau complet construit sur un ensemble $X_{n}$ à $n$ sommets, et soit $c$ un vecteur Coût défini sur les arcs de $K_{n}$ et satisfaisant l'Inégalité Triangulaire. On suppose que $A$ est l'ensemble de tous les arcs de $K_{n}$, que $K$ est l'ensemble de tous les couples de sommets de $K_{n}$, que $p=0$, et que les capacités $M A X, C$ min et $C \max$ sont respectivement infinies, nulles et infinies. On suppose enfin que chaque flot $f(x, y), x, y$ dans $X_{n}$, doit exprimer l'acheminement d'une quantité $1 / n^{2}$ de flux depuis $x$ vers $y$. Pour une telle instance de CFEMF-OD, on a :

- $V$ est la longueur pour $c$ d'une plus courte tournée du Voyageur de Commerce dans $K_{n}$;

- $B=D=V=$ valeur optimale de CFEMF-OD;

- $V^{*}$ est seulement égale à la valeur moyenne du coût d'un arc.

\subsection{Comparaison thÉorique des 3 schÉmas DRCOUP, DRFLOT ET DME}

Théorème 1. On a toujours $B \geq V^{*}$ et $D \geq V^{*}$. Si de plus $M A X=+\infty$, alors on $a B=D$.

Démonstration. Pour tout vecteur $\lambda \geq 0$, indexé sur $A$, notons $V_{\lambda}$ la valeur optimale du programme $\mathbf{P}^{*}$-Coup $_{\lambda}$ obtenu à partir de $\mathbf{C F E M F}^{*}$ par relaxation lagrangienne de la contrainte de couplage (C3). Nous savons (théorie de la dualité) que $V=\operatorname{Sup}_{\lambda \geq 0} V_{\lambda}$. De l'inégalité $V_{\lambda} \leq B_{\lambda}$, nous déduisons $V \leq B$. Le même raisonnement fournit $V \leq D$.

Supposons à présent $M A X=+\infty$. Dans ce cas, nous savons (Eq. (E2)) que $B=\operatorname{Sup}_{\lambda \geq 0} B_{\lambda}$ s'écrit aussi :

$B=\operatorname{Sup}_{\Pi \text { tels que } \Pi . M(G)_{\leq c}} \operatorname{In} f_{f \text { tels que } C \min \leq f \leq C \max }(p . \operatorname{Sum}(f)+$

$$
\left(c-\Pi \cdot M(G)_{A} \cdot\left\lceil\operatorname{Sum}(f)_{A}\right\rceil\right) .
$$

On a aussi (Eq. (E3)) : $D=\operatorname{Sup}_{\mu}$ tels que $c-\mu M_{\geq 0}$ Inf $f \geq 0$ compatibles $C \min$ et $C$ max $\left((c-\mu \mathrm{M})_{A}\right.$. $\left.\left\lceil\operatorname{Sum}(f)_{A}\right\rceil+p . \operatorname{Sum}(f)\right)$. On voit dès lors que $B$ et $D$ coincident.

Nous allons à présent montrer que les cas où les trois schémas de résolution DME, DRCOUP et DRFLOT produisent un résultat optimal sont les mêmes. Nous introduisons pour ce faire les définitions suivantes :

Configuration d'arrêt pour le schéma DME.

Soit $\left(F^{*}, f^{*}\right)$ une solution réalisable de $P$, telle que $F^{*}$ soit optimal pour $\mathbf{C F E M F}_{f *}$. Soient $\mu^{*}, \tau^{*}, \lambda^{*}$ trois vecteurs respectivement indexés sur $X$, sur $E$ et sur $A$, et optimaux pour le Dual $\mathbf{D U}_{f^{*}}$ de $\mathbf{C F E M F} f_{f^{*}}$.

Nous disons que $\left(F^{*}, f^{*}\right)$ et $\left(\mu^{*}, \tau^{*}, \lambda^{*}\right)$ sont une configuration d'arrêt du schéma DME appliqué à CFEMF si $f^{*}$ est solution optimale du problème $\operatorname{P-aux}\left(\lambda^{*}\right)$ :

$\{$ Trouver un multiflot $f$, compatible avec $C \min$ et $C \max$, et qui minimise $\left.p . \operatorname{Sum}(f)+\lambda^{*} .\left\lceil\operatorname{Sum}(f)_{A}\right\rceil\right\}$. 
Configuration d'arrêt pour le schéma DRCOUP.

Soient $\lambda^{*} \geq 0$ un vecteur indexé sur $E$ et nul sur $A$, et $\left(F^{*}, f^{*}\right)$ une solution optimale de P-Coup $\mathbf{P}_{\lambda *}$. Nous disons que $\left(F^{*}, f^{*}\right)$ et $\lambda^{*}$ sont une configuration d'arrêt du schéma DRCOUP appliqué à CFEMF si, pour tout $\lambda$ dans $\Lambda=\{\lambda \geq 0$, indexés sur $E$, nuls sur $E-A$, et tels qu'il n'existe pas de circuit négatif pour le coût $(c-\lambda)$ dans le réseau $\left.\left(X, E^{\infty}\right)\right\}$, on a : $\left(\lambda-\lambda^{*}\right) \cdot\left(F^{*}-\left\lceil\operatorname{Sum}\left(f^{*}\right)\right\rceil \geq 0\right.$.

\section{Configuration d'arrêt pour le schéma DRFLOT.}

Soient $\mu^{*}$ un vecteur indexé sur $X$ et $\left(F^{*}, f^{*}\right)$ une solution optimale du problème $\mathbf{P}-$ Flot $_{\mu^{*}}$. $\left(F^{*}, f^{*}\right)$ et $\mu^{*}$ sont une configuration d'arrêt du schéma DRFLOT appliqué à CFEMF si, pour tout $\mu$ dans $\Omega=\{\mu$ indexés sur $X$ tels que $\left.(c-\mu \cdot M(G))_{E \infty 8} \geq 0\right\}$, on a l'inégalité : $(\mu-$ $\left.\left.\mu^{*}\right) \cdot M(G)\right)_{A} \cdot\left\lceil\operatorname{Sum}\left(f^{*}\right)_{A}\right\rceil \geq 0$.

Théorème 2. Les énoncés suivants sont équivalents :

1. il existe une configuration d'arrêt pour le schéma $\mathbf{D M E}$;

2. il existe une configuration d'arrêt pour le schéma DRCOUP;

3. il existe une configuration d'arrêt pour le schéma DRFLOT;

4. $V=B$;

5. $V=D$.

Démonstration. De (1) déduisons (2), (3), (4) et (5). Soit (notations ci-dessus) une configuration d'arrêt $\left(F^{*}, f^{*}\right),\left(\mu^{*}, \tau *, \lambda^{*}\right)$ du schéma DME. Nous pouvons supposer que $\lambda^{*}$ est en fait indexé sur $E$ et que $\lambda_{E-A}^{*}=0$. Par dualité, il vient : $(c-\lambda *) \cdot F^{*}+\lambda^{*} \cdot\left\lceil\operatorname{Sum}\left(f^{*}\right)\right\rceil+p \cdot \operatorname{Sum}\left(f^{*}\right)=V_{f^{*}}$.

$F^{*}$ est donc solution optimale du programme :

$\left\{\right.$ Chercher un flot $F$, entier, tel que $0 \leq F \leq M A X$ et minimisant c.F $\left.-\lambda^{*} . F\right\}$.

Pour tout $\lambda$ dans $R^{+E}$ tel que $\lambda_{E-A}=0$, on a $:\left(c-\lambda^{*}\right) \cdot F^{*}+\lambda^{*} \cdot\left\lceil\operatorname{Sum}\left(f^{*}\right)\right\rceil \geq$ $(c-\lambda) \cdot F^{*}+\lambda .\left\lceil\operatorname{Sum}\left(f^{*}\right\rceil\right.$.

Le couple $\left(F^{*}, f^{*}\right)$ étant solution réalisable de $\mathbf{P}-$ Coup $_{\lambda *}$, il découle de (E6) et du fait que $f^{*}$ est solution optimale de $\mathbf{P}$-aux $\left(\lambda^{*}\right)$, que $\left(F^{*}, f^{*}\right)$ sont une solution optimale de P-Coup - $_{\lambda_{*}}$. De $V_{f^{*}} \geq V \geq B \geq B_{\lambda^{*}}$, on déduit alors que $V=B$. Et de (E7) on déduit que $\left(F^{*}, f^{*}\right)$ et $\lambda^{*}$ sont une configuration d'arrêt de DRCOUP (Points (4) et (2)).

De même, on voit que $\left(c-\mu^{*} \cdot M(G)\right) \cdot F^{*}+p \cdot \operatorname{Sum}\left(f^{*}\right)=V_{f^{*}}$. Le vecteur $F^{*}$ est donc solution optimale du programme : \{Chercher $F$, entier, tel que $F_{A} \geq$ $\operatorname{Sum}\left(f^{*}\right)_{A}$, et $F \leq \mathrm{MAX}$, et minimisant c.F $\left.-\mu^{*} \cdot M(G) . F\right\}$.

$(\mathrm{E} 8)$.

On voit aussi que pour tout vecteur $\mu$, indexé sur $X$, on a : $\mu^{*} \cdot M(G) \cdot F^{*} \leq$ $\mu . M(G) . F^{*}$.

De (E9) on déduit $M(G) \cdot F^{*}=0$ et de $(\mathrm{E} 8)$ on déduit :

- si $e$ est dans $A$ et $\left(c-\mu^{*} \cdot M(G)\right)_{e} \geq 0$, alors $F_{e}^{*}=\left[\operatorname{Sum}\left(f^{*}\right)_{e}\right]$;

- si $e$ n'est pas dans $A$ et si $\left(c-\mu^{*} \cdot M(G)\right)_{e} \geq 0$, alors $F_{e}^{*}=0$;

- si $\left(c-\mu^{*} \cdot M(G)\right)_{e}<0$ alors $F_{e}^{*}=M A X_{e}$. 
On en déduit que $\left(c-\mu^{*} \cdot M(G)\right) \cdot F^{*}+p \cdot \operatorname{Sum}\left(f^{*}\right)=p \cdot \operatorname{Sum}\left(f^{*}\right)+(c-$ $\mu * . M(G))_{A}^{+} \cdot\left\lceil\operatorname{Sum}\left(f^{*}\right)_{A}\right\rceil+\left(c-\mu^{*} \cdot M(G)\right)^{-} \cdot M A X$.

Le triplet $\left(\mu *, \tau *, \lambda^{*}\right)$ vérifie aussi $\mu^{*} . M(G)-\tau^{*}+\lambda^{*} \leq c$, et maximise, sous cette condition, la quantité $\tau^{*} . M A X+\lambda^{*}$. $\left.\left\lceil\operatorname{Sum}\left(f^{*}\right)\right]\right\rceil$. On déduit $\left(c-\mu^{*} \cdot M(G)\right) \cdot F^{*}+$ $p \cdot \operatorname{Sum}\left(f^{*}\right)=p \cdot \operatorname{Sum}\left(f^{*}\right)+\lambda^{*} \cdot\left[\operatorname{Sum}\left(f^{*}\right)_{A}\right]+\tau^{*} \cdot M A X$, et, puisque $f^{*}$ est solution optimale de $\mathbf{P}-\mathbf{a u x}\left(\lambda^{*}\right)$, que le couple $\left(F^{*}, f^{*}\right)$, qui est déjà solution réalisable de P-Flot $_{\mu^{*}}$, en est aussi une solution optimale. De $V_{f *} \geq V \geq D \geq D_{\mu^{*}}$, on déduit alors $V=D$. Et de (E9) on déduit que $\left(F^{*}, f^{*}\right)$ et $\lambda^{*}$ définissent une configuration d'arrêt du schéma DRFLOT (points (5) et (3)).

De (3) déduisons (5).

Soit une configuration d'arrêt $\left(F^{*}, f^{*}\right)$ et $\mu^{*}$ pour le schéma DRFLOT. $F^{*}$ et $f^{*}$ sont donc une solution optimale de P-Flot $\mu^{*}$ et $\mu^{*}$ est solution optimale du programme $H$ :

$\left\{\right.$ Trouver $\mu$, indexé sur $X$, tel que $(c-\mu \cdot M(G))_{E \infty} \geq 0$, et qui minimise $\left.\mu \cdot M(G) \cdot F^{*}\right\}$. Le dual $H^{*}$ de $H$ s'écrit :

$\left\{\right.$ Trouver $z \geq 0$, indexé sur $E$, nul sur $E-E^{\infty}$, tel que $M(G) . z+M(G) \cdot F^{*}=0$, et minimisant c.z\};

et admet une solution optimale entière $z^{*}$. Le flot $F 1=F^{*}+z^{*}$ est entier, tel que $M A X \geq F$ et que $F 1_{A} \geq\left[\operatorname{Sum}\left(f^{*}\right)_{A}\right]$. On a aussi c.F1 $+p \cdot \operatorname{Sum}\left(f^{*}\right)=$ $c . F^{*}+c . z *+p \cdot \operatorname{Sum}\left(f^{*}\right)=\left(c-\mu^{*} \cdot M(G)\right) \cdot F^{*}+p \cdot \operatorname{Sum}\left(f^{*}\right)=D_{\mu^{*}} . F 1$ et $f^{*}$ forment donc une solution réalisable de CFEMF, optimale puisque $D_{\mu *} \leq D \leq V$. On déduit $D=V($ point $(5))$.

De (2) déduisons (4).

Soit une configuration d'arrêt $\left(F^{*}, f^{*}\right), \lambda^{*}$ pour le schéma DRCOUP. $F^{*}$ et $f^{*}$ sont donc une solution optimale du programme P-Coup ${ }_{\lambda *}$ et $\lambda^{*}$ est solution optimale du programme $K$ suivant :

$\{$ Trouver $\lambda \geq 0$, indexé sur $E$ et nul $E$ - $A$, et $\mu$, indexé sur $X$, tels que $(\mu \cdot M(G)+$ $\lambda)_{E \infty} \leq c_{e}$, et qui maximisent $\left.\lambda .\left(\left\lceil\operatorname{Sum}\left(f^{*}\right)\right\rceil-F^{*}\right)\right\}$.

Le dual $K^{*}$ de $K$ s'écrit : \{Trouver un flot $u \geq 0$, indexé sur $E$ et dont la trace sur $E-E^{\infty}$ est nulle, tel que :

$u_{e} \geq\left(\left\lceil\operatorname{Sum}\left(f^{*}\right)\left\lceil-F^{*}\right)_{e}\right.\right.$ pour tout arc e dans $A \cap E^{\infty} ;(E 10)$

et qui minimise la quantité c.u\}.

Soit $u^{*}$ une solution optimale, que l'on peut choisir entière, de $K^{*}$. Le flot $F 1 *=$ $F^{*}+u^{*}$ est entier, compatible avec le vecteur $M A X$. Si $e \in A \cap E^{\infty}$, alors on déduit de (E10) que $F 1_{e} \geq\left\lceil\operatorname{Sum}\left(f^{*}\right)_{e}\right\rceil$. Dans le cas où $e \in A \cap E^{\infty}$, alors le programme $K$ n'est borné (et n'admet donc $\lambda^{*}$ comme solution optimale) que si $F_{e}^{*} \geq\left\lceil\operatorname{Sum}\left(f^{*}\right)_{e}\right\rceil$.

Le couple $\left(F 1, f^{*}\right)$ est donc une solution réalisable de CFEMF. On a enfin que :

$$
\begin{aligned}
c . F 1+p . \operatorname{Sum}\left(f^{*}\right)=c . F^{*}+c . u *+p . \operatorname{Sum}( & \left.f^{*}\right)=(\text { Dualité }) c . F^{*}-\lambda^{*} . F^{*} \\
& +\lambda^{*} \cdot \operatorname{Sum}\left(f^{*}\right)+p \cdot \operatorname{Sum}\left(f^{*}\right)=B_{\lambda^{*}} \leq V .
\end{aligned}
$$

Il s'ensuit que $B_{\lambda^{*}}=B=V$, c'est-à-dire le point (4). 
De (4), déduisons (1).

Supposons donc $B=V$. L'ensemble des couples $(F, f)$ susceptibles d'être solution optimale d'un problème $\mathbf{P}-\mathbf{C o u p}_{\lambda}$ peut être considéré comme réduit à l'ensemble des sommets d'un certain polyèdre et donc comme fini. Il en découle l'existence d'un vecteur $\lambda^{*} \geq 0$, qui est indexé sur $E$ et de trace nulle sur $E-A$, et d'un couple $\left(F^{*}, f^{*}\right)$, solution optimale de CFEMF, tels que $\left(F^{*}, f^{*}\right)$ est aussi solution optimale du programme P-coup $\lambda_{\lambda^{*}}$. On a alors $\lambda^{*} .\left(F^{*}-\left\lceil\operatorname{Sum}\left(f^{*}\right)\right\rceil\right)=0$. Soient $\mu^{*}$ un vecteur indexé sur $X$, et $\tau^{*} \geq 0$ un vecteur indexé sur $E$ qui constituent une solution duale optimale du programme :

$\{$ Trouver un flot $F$ (entier) $\geq 0$, tel que $F \leq M A X$ et qui minimise la quantité $\left.\left(c-\lambda^{*}\right) \cdot F\right\}$.

Le triplet $\left(\mu^{*}, \tau^{*}, \lambda^{*}\right)$ est alors solution réalisable du dual $\mathbf{D U}_{f^{*}}$ de $\mathbf{C F E M F} f_{f^{*}}$, telle que :

$$
c . F^{*}-\left(\lambda^{*}\left(\left\lceil\operatorname{Sum}\left(f^{*}\right)\right\rceil\right)-\tau^{*} \cdot M A X\right)=\left(c-\lambda^{*}\right) . F^{*}+\tau^{*} \cdot M A X=0,
$$

et donc en fait solution optimale de $\mathbf{D} \mathbf{U}_{f *}$. Le fait que $f^{*}$ soit alors solution optimale du programme :

$\{$ Trouver un multiflot $f \geq 0$, tel que $C \min \leq f \leq C \max$ et minimisant $p . \operatorname{Sum}(f)+$ $\left.\lambda^{*} \cdot \operatorname{Sum}(f)\right\}$,

induit alors que $\left(F^{*}, f^{*}\right)$ et $(\mu *, \tau *, \lambda *)$ sont une configuration d'arrêt pour le schéma DME (point (1)).

De (5) déduisons (1). L'égalité $D=V$ implique l'existence de $\mu^{*}$, tel que $\mathbf{P}$ Flot $_{\mu^{*}}$ admet une même solution optimale $\left(F^{*}, f^{*}\right)$ que CFEMF. On a alors : $\mu * . M(G) \cdot F^{*}=0$. Soit le programme :

$\left\{\right.$ Trouver $F \geq 0$, indexé sur $E$, tel que $F_{A} \geq\left\lceil\operatorname{Sum}\left(f^{*}\right)_{A}\right\rceil$ et $F \leq M A X$ et minimisant $\left.\left(c-\mu^{*} \cdot M(G)\right) . F\right\}$.

Ce programme admet $F^{*}$ comme solution optimale à laquelle correspond donc une solution duale optimale associée $\left(\tau^{*}, \lambda^{*}\right)$. Les vecteurs $\tau^{*} \geq 0$ et $\lambda^{*} \geq 0$ sont indexés sur $E$ et tels que $\lambda_{E-A}^{*}$ est nulle, et $\mu^{*}, \tau^{*}$ et $\lambda^{*}$ définissent alors une solution optimale du dual $\mathbf{D U}_{f *}$ de $\mathbf{C F E M F}_{f *}$. On peut en fait choisir $\tau^{*}$ et $\lambda^{*}$ tels que :

$$
\tau *=\left(\mu^{*} \cdot M(G)-c\right)^{+} \text {et }\left(\lambda^{*}\right)_{A}=\left(c-\mu^{*} \cdot M(G)\right)_{A}^{+} .
$$

Donc, si $f$ est tel que $C \min \leq f \leq C \max$ et si $F(f)$ est solution optimale du programme $\left(\mathbf{P}-\text { Flot }_{\mu^{*}}\right)_{f}$, alors la quantité $c . F(f)+p \cdot \operatorname{Sum}(f)-\mu^{*} \cdot M . F(f)$ s'écrit $\lambda^{*} \cdot \operatorname{Sum}(f)+p \cdot \operatorname{Sum}(f)-\tau^{*} \cdot M A X$. Il s'ensuit que $f^{*}$ est solution optimale du problème $\mathbf{P}$-aux $\left(\lambda^{*}\right)$ et donc que $\left(F^{*}, f^{*}\right)$ et $\left(\mu *, \tau^{*}, \lambda^{*}\right)$ sont une configuration d'arrêt de DME (point (1)). 


\subsection{UNE ILLUSTRATION DES THÉORÈMES 1 ET 2}

Considérons le réseau $G=(X, E)$ suivant :

$$
\begin{aligned}
& X=\{A, B, C, D\} ; \\
& E=\{[A, B],[B, A],[B, C],[C, A], \\
& \quad[C, D],[D, C],[D, A],[A, D],[A, C],[B, D]\} ; A=E .
\end{aligned}
$$

\begin{tabular}{|c|c|c|c|c|c|c|c|c|c|c|}
\hline & {$[A, B]$} & {$[B, A]$} & {$[B, C]$} & {$[C, B]$} & {$[C, D]$} & {$[D, C]$} & {$[D, A]$} & {$[A, D]$} & {$[A, C]$} & {$[B, D]$} \\
\hline$c$ & 1 & 0 & 1 & 0 & 1 & 0,2 & 0,1 & 4 & 2,5 & 2,5 \\
\hline
\end{tabular}

Chaque arc $e$ dans $E$ est pourvu d'un coût $p_{e}=0,5$, ainsi que d'un coût $c_{e}$ défini par le tableau suivant : $k=1 \ldots 2\} \geq 0$ tels que :

- le flot $f(1)$ exprime l'acheminement d'une quantité de flot 0,4 de $A$ vers $C$;

- le flot f(2) exprime l'acheminement d'une quantité de flot 0,6 de B vers D;

- pour tout arc e dans $A$, on a $F_{e} \geq \operatorname{Sum}(f)_{e}$; et qui minimise la quantité c.F $+p \cdot(f(1)+f(2))$.

Nous voyons qu'une solution optimale du problème ainsi posé est définie par :

- un flot $F^{*}$ valant 1 sur les arcs du circuit $(A, B, C, D, A)$ et 0 ailleurs;

- un flot $f^{*}(1)$ valant 0,4 sur les arcs du chemin $(A, B, C)$;

- un flot $f^{*}(2)$ valant 0,6 sur les arcs du chemin $(B, C, D)$;

et que la valeur optimale associée est $3,1+2=5,1$.

Le couple $\left(F 1 *=0, f 1 *=f^{*}\right)$ et le vecteur $\lambda^{*}$ ci-dessous sont une configuration d'arrêt du schéma DRCOUP.

\begin{tabular}{|ccccccccccc|}
\hline & {$[\mathrm{A}, \mathrm{B}]$} & {$[\mathrm{B}, \mathrm{A}]$} & {$[\mathrm{B}, \mathrm{C}]$} & {$[\mathrm{C}, \mathrm{B}]$} & {$[\mathrm{C}, \mathrm{D}]$} & {$[\mathrm{D}, \mathrm{C}]$} & {$[\mathrm{D}, \mathrm{A}]$} & {$[\mathrm{A}, \mathrm{D}]$} & {$[\mathrm{A}, \mathrm{C}]$} & {$[\mathrm{B}, \mathrm{D}]$} \\
$\lambda^{*}$ & 1 & 0 & 1 & 0 & 1,1 & 0,1 & 0 & 4,1 & 2,5 & 2,6 \\
\hline
\end{tabular}

La valeur $B$ alors obtenue est elle aussi égale à 5,1 . Nous sommes ici dans les conditions du théorème 1 , et donc $D=5,1$. Le couple $\left(\mathrm{F} 1^{*}, \mathrm{f} 1^{*}\right)$ et le vecteur $\mu^{*}$ ci-dessous sont une configuration d'arrêt pour le schéma DRFLOT.

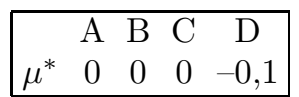

Testons à présent le comportement sur cet exemple du schéma DME. À l'intérieur des tableaux ci-dessous, $\lambda$ et $\mu$ désignent les composantes du vecteur dual, pour le problème $\mathbf{C F E M F}_{f}$, qui sont respectivement indexées sur $E$ et sur $X$. Les flots $f(1)$ et $f(2)$ sont initialisés de façon à minimiser la quantité $p \cdot(f(1)+f(2))$, 
et sont remis à jour à chaque itération par minimisation de la quantité $\lambda .[(f(1)+$ $f(2))]+p \cdot(f(1)+f(2))$. Nous obtenons :

$1^{\text {re }}$ itération :

$f(1)$ est associé au chemin $(A, C) ; f(2)$ est associé au chemin $(B, D)$;

$F$ est associé au circuit $(A, C, B, D, C, D, A) ; \mu$ est défini par le tableau :

$$
\begin{array}{|cccc|}
\hline \mathrm{A} & \mathrm{B} & \mathrm{C} & \mathrm{D} \\
0 & 1 & -1,1 & -0,1 \\
\hline
\end{array}
$$

$\lambda$ est défini par le tableau :

\begin{tabular}{|cccccccccc|}
\hline$[\mathrm{A}, \mathrm{B}]$ & {$[\mathrm{A}, \mathrm{C}]$} & {$[\mathrm{A}, \mathrm{D}]$} & {$[\mathrm{B}, \mathrm{A}]$} & {$[\mathrm{B}, \mathrm{C}]$} & {$[\mathrm{B}, \mathrm{D}]$} & {$[\mathrm{C}, \mathrm{B}]$} & {$[\mathrm{C}, \mathrm{D}]$} & {$[\mathrm{D}, \mathrm{A}]$} & {$[\mathrm{D}, \mathrm{C}]$} \\
0 & 3,6 & 4,1 & 1 & 3,1 & 3,6 & 0 & 0 & 0 & 1,2 \\
\hline
\end{tabular}

\section{$2^{e}$ itération:}

$f(1)$ est associé au chemin $(A, B, D, C) ; f(2)$ est associé au chemin $(B, D)$; $F$ est associé au circuit $(A, B, D, C, C, B, A) ; \mu$ est défini par le tableau :

$$
\begin{array}{|cccc|}
\hline \mathrm{A} & \mathrm{B} & \mathrm{C} & \mathrm{D} \\
0 & 0 & 0 & 1 \\
\hline
\end{array}
$$

$\lambda$ est défini par le tableau :

\begin{tabular}{|cccccccccc|}
\hline$[\mathrm{A}, \mathrm{B}]$ & {$[\mathrm{A}, \mathrm{C}]$} & {$[\mathrm{A}, \mathrm{D}]$} & {$[\mathrm{B}, \mathrm{A}]$} & {$[\mathrm{B}, \mathrm{C}]$} & {$[\mathrm{B}, \mathrm{D}]$} & {$[\mathrm{C}, \mathrm{B}]$} & {$[\mathrm{C}, \mathrm{D}]$} & {$[\mathrm{D}, \mathrm{A}]$} & {$[\mathrm{D}, \mathrm{C}]$} \\
1 & 2,5 & 3 & 0 & 1 & 1,5 & 0 & 0 & 1,1 & 1,2 \\
\hline
\end{tabular}

\section{$3^{e}$ itération :}

$f(1)$ est associé au chemin $(A, B, C) ; f(2)$ est associé au chemin $(B, C, D)$; $F$ est associé au circuit $(A, B, C, D) ; \mu$ est défini le tableau :

$$
\begin{array}{|cccc|}
\hline A & B & C & D \\
0 & 0,1 & 0,1 & -0,1 \\
\hline
\end{array}
$$

$\lambda$ est défini par le tableau :

\begin{tabular}{|cccccccccc|}
\hline$[\mathrm{A}, \mathrm{B}]$ & {$[\mathrm{A}, \mathrm{C}]$} & {$[\mathrm{A}, \mathrm{D}]$} & {$[\mathrm{B}, \mathrm{A}]$} & {$[\mathrm{B}, \mathrm{C}]$} & {$[\mathrm{B}, \mathrm{D}]$} & {$[\mathrm{C}, \mathrm{B}]$} & {$[\mathrm{C}, \mathrm{D}]$} & {$[\mathrm{D}, \mathrm{A}]$} & {$[\mathrm{D}, \mathrm{C}]$} \\
0,9 & 2,4 & 4,1 & $-0,1$ & 1 & 2,6 & 0 & 1,2 & 0 & 0 \\
\hline
\end{tabular}

$4^{e}$ itération $:(F, f)$ et $(\mu, \lambda)$ définissent une configuration d'arrêt.

\section{Comment traiter le problème auxiliaire P-Aux?}

Il s'agit ici de spécifier l'instruction (I1) du schéma DME, et décrire quel traitement apporter au problème $\mathbf{P}$-aux :

$\mathrm{P}-\mathbf{a u x}(\lambda):\{$ Étant donnés le réseau $G=(X, E)$, les familles $C \mathrm{~min}$ et $C \max$, les vecteurs $\lambda \geq 0$ et $p \geq 0$, on cherche un multiflot $f=\{f(k), k \in$ $K\}$, tel que $C \min \leq f \leq C \max$ et minimisant $\lambda$. $\lceil\operatorname{Sum}(f)\rceil+p . \operatorname{Sum}(f)\}$. 
Ce problème peut bien sûr être reformulé en introduisant un vecteur auxiliaire $z$, entier et dominant $\operatorname{Sum}(f)$, et en imposant la minimisation de $\lambda . z+p \cdot \operatorname{Sum}(f)$. Ce faisant, on va toutefois en sens inverse du processus amorcé, qui vise à traiter $\operatorname{Sum}(f)$ comme objet principal et à le gérer en tirant parti de la structure des espaces de flots.

Afin de poursuivre dans le sens suggéré par les sections précédentes, nous devons procéder en deux temps :

- nous devons d'abord disposer (Sect. 4.1), d'un outil nous permettant de gérer la quantité $\lambda$. $\lceil\operatorname{Sum}(f)\rceil+p \cdot \operatorname{Sum}(f)$, en agissant directement sur $\operatorname{Sum}(f)$, ou, si l'ensemble $K$ est réduit, sur un des composants de $f$;

- nous devons ensuite (Sect. 4.2), gérer l'articulation entre $\operatorname{Sum}(f)$ et les composants de $f$. Le but est ici d'éviter de n'agir sur $\operatorname{Sum}(f)$ qu'au travers de ses composantes prises de façon séparée. Dans le cas où $K$ est grand et où les valeurs de chaque composante $f(k)_{e}$ ont vocation à être petites par rapport à l'unité, (ce qui est le cas pour les problèmes de la forme CFEMF-OD et pour les problèmes exemples de la Sect. 2), alors une perturbation de $f$, localisée sur une seule composante, risque en effet de n'induire rapidement aucune modification du vecteur $\lceil\operatorname{Sum}(f)\rceil$, et donc aucune amélioration de la quantité $\lambda$. $\lceil\operatorname{Sum}(f)\rceil+p . \operatorname{Sum}(f)\}$.

\subsection{La Gestion de La Quantité $\lambda$. $\lceil\operatorname{Sum}(f)\rceil+p$.Sum $(f)$ : LE Problème FCEM}

Il s'agit donc du problème $\mathbf{P}$-aux considéré dans le cas où le multiflot $f$ est en fait un flot:

Problème de Flot de Cô̂t Entier Minimum (FCEM) : \{Étant donnés le réseau $G=(X, E)$, 2 vecteurs capacités $C \min$ et $C \max$, deux vecteurs coûts $\lambda$ et $p$, positifs ou nuls, tous indexés sur $E$, un vecteur flot $u$, on cherche alors un flot $f \geq 0$, tel que $C \min \leq f \leq C \max$, et qui minimise : p.Sum $(f) b+\lambda .\lceil(f+u)\rceil\}$.

Proposition 2. Le problème $\boldsymbol{F C E M}$ est NP-Difficile.

Démonstration. Considérons le problème PLO dit de Localisation, connu pour être NP-complet (voir KHU72) :

Problème P-LO de Localisation : \{Étant donnés un ensemble $Z$, un vecteur "Cô̂t de Construction" $\alpha$ indexé sur Z, un vecteur "Coût de Distribution" $\beta$, indexé sur X.X, un nombre "Seuil" $S$, on cherche un sousensemble $A$ de $Z$, tel que $\Sigma_{x \text { dans } A} \alpha_{x}+\Sigma_{y \text { dans } X}$ Inf $x$ dans $A \beta_{x, y} \leq$ $S\}$.

À une instance $(Z, S, \alpha, \beta)$ de ce problème, associons le réseau $G=(X, E)$ suivant :

- $X=\{$ DEBUT, FIN $\} \cup Z \cup Z^{\prime}$, où $Z^{\prime}$ est une copie de $Z$ et où DEBUT et FIN sont deux sommets additionnels. Pour chaque élément $x$ de $Z$, nous notons $x^{\prime}$ son homologue dans $Z^{\prime}$;

- $E=\{[$ FIN, DEBUT $]\} \cup\left\{\left[\right.\right.$ DEBUT, $\left.\left.x^{\prime}\right], x^{\prime} \in Z^{\prime}\right\} \cup\left\{\left[x^{\prime}, y\right], x^{\prime}\right.$ dans $Z^{\prime}$, $y \in Z\} \cup\{(x$, FIN $), x \in X\}$. 
Résoudre P-LO sur l'instance $(Z, S, \alpha, \beta)$ revient à trouver sur $G$ un flot $f \geq 0$, tel que $f([x, \mathrm{FIN}])=1 / \operatorname{Card}(\mathrm{Z})$ pour tout $x$ dans $Z$, et tel que : $\Sigma_{x \text { dans } Z} \alpha_{x}$. $f\left(\left[\right.\right.$ DEBUT, $\left.\left.x^{\prime}\right]\right)+\Sigma_{x, y \text { dans } Z} \beta_{x, y} \cdot f\left(\left[x^{\prime}, y\right]\right) \leq S$. Le résultat en découle.

\section{Cycles généralisés améliorants pour le problème FCEM}

Considérons à présent une instance du problème FCEM, conformément aux notations ci-dessus. Soient $f$ une solution réalisable de FCEM, $\gamma$ un cycle de $G$ et $q>0$ un nombre. Nous posons :

$E(\gamma,+)=$ ensemble des arcs de $\gamma$ orientés dans le même sens que $\gamma$;

$E(\gamma,-)=$ ensemble des arcs de $\gamma$ orientés dans le sens opposé au sens de $\gamma$; et, pour tout $\operatorname{arc} e$ :

$$
\begin{aligned}
& D_{f}(e, q)=q \cdot p_{e}+\lambda_{e} \cdot\left[\left\lceil(f+u)_{e}+q\right\rceil-\left\lceil(f+u)_{e}\right\rceil\right] ; \\
& D_{f}^{*}(e, q)=-q \cdot p_{e}-\lambda_{e} \cdot\left[\left\lceil(f+u)_{e}\right\rceil-\left\lceil(f+u)_{e}-q\right\rceil\right] .
\end{aligned}
$$

Le couple $(\gamma, q)$, dit Cycle Généralisé, sera améliorant, au sens du problème FCEM, pour le flot courant $f$, si :

- pour tout $e$ dans $E(\gamma,+), f_{e}+q \leq C \max _{e}$; pour tout $e$ dans $E(\gamma,-)$, $f_{e}-q \geq C \min _{e}$

- longueur $(\gamma) \geq 3 ;($ Contrainte de Longueur $)$

$-\Sigma_{e \text { dans } E(\gamma,+)} D_{f}(e, q)+\Sigma_{e \text { dans } E(\gamma,-)} D_{f}^{*}(e, q)<0$.

Clairement, l'existence d'un Cycle Généralisé Améliorant permet de remplacer la solution courante $f$ par $f+q \cdot \phi_{\gamma}$, et d'améliorer $f$ sans violer de contrainte. La recherche d'un tel cycle généralisé améliorant constituera donc l'épine dorsale de la procédure de traitement de FCEM que nous allons proposer ici. Dans cette perspective, le sens de la contrainte de Longueur ci-dessus est qu'un $\operatorname{arc} e$ de $G$ peut être tel que $D_{f}(e, q)+D_{f}^{*}(e, q)<0$, mais qu'il n'est évidemment pas possible de simultanément augmenter et diminuer la valeur de $f$ sur un tel arc.

Remarque. L'absence d'un Cycle Généralisé Améliorant $(\gamma, q)$ ne signifie pas que le flot courant $f$ soit une solution optimale pour le problème FCEM associé. Considérons en effet l'exemple suivant :

$$
\begin{aligned}
& X=\{a, b, c, d\} ; E=\{[a, b],[b, c],[c, a],[b, d],[d, a]\} \\
& p([a, b])=0 ; p(e)=1 \text { pour tout arc } e \text { autre que }[a, b] ; \\
& u=0 ; \lambda([a, b])=-10 ; \lambda(e)=0 \text { pour tout arc } e \text { autre que }[a, b] \\
& C \min =0 ; C \max ([a, b])=4 ; C \max (e)=1,6 \text { pour tout arc } e \text { autre que } \\
& {[a, b] ;} \\
& f([a, b])=2,2 ; f(e)=1,1 \text { pour tout arc } e \text { autre que }[a, b] .
\end{aligned}
$$

Ce flot $f$ n'est pas optimal pour le problème FCEM, alors que n'existe aucun cycle généralisé améliorant $f$.

La recherche du couple $(\gamma, q)$ peut se reformuler en termes de circuits négatifs dans un réseau auxiliaire $G_{f}^{*}=\left(X, E^{*}\right)$, muni d'une fonction coût $C_{f}$, dite de Coût Généralisé, définis comme suit :

- $E^{*}=\left\{e, e^{-1}, e\right.$ dans $\left.E\right\} ;$ (si $e$ est un arc $[x, y]$ dans $E, e^{-1}$ désigne l'arc symétrique $[y, x])$. 
- Pour $e$ dans $E$, et $q>0$, on pose :

$-s(f, e)=C \max _{e}-f_{e} ; s\left(f, e^{-1}\right)=f_{e}-C \min _{e} ;$

- $t(f, e)=\left\lceil f_{e}+u_{e}\right\rceil-\left(f_{e}+u_{e}\right) ; t\left(f, e^{-1}\right)=\left(f_{e}+u_{e}\right)-\left\lceil f_{e}+u_{e}\right\rceil ;$

- $C_{f}(q, e)=q \cdot p_{e}+\lceil(q-t(e))\rceil \cdot \alpha_{e}$, si $q \leq s(e)$ et $C_{f}(q, e)=+\infty$ (un grand nombre) sinon;

$-C_{f}\left(q, e^{-1}\right)=-q \cdot p_{e}-\left\lfloor 1+\left(q-t\left(e^{-1}\right)\right)\right\rfloor \cdot \alpha_{e}, \operatorname{si} q \leq s\left(e^{-1}\right)$ et $C_{f}\left(q, e^{-1}\right)=$ $+\infty$ sinon.

La recherche du couple $(\gamma, q)$ devient alors celle d'un circuit élémentaire $\Gamma$ dans $G *_{f}$, de longueur $\geq 3$, et d'un nombre $q>0$, tels que $: L_{f}(\Gamma, q)=\Sigma_{e * \text { dans } \Gamma}$ $C_{f}\left(q, e^{*}\right)<0$. Un tel couple $(\Gamma, q)$ sera un Circuit Négatif Généralisé.

\section{Recherche d'un Circuit Négatif Généralisé dans le réseau G*}

Nous avons d'abord besoin d'un outil de filtrage nous permettant d'identifier les valeurs $q$ qui sont susceptibles de faire partie d'un Circuit Négatif Généralisé $(\Gamma, q)$ de $G *_{f}$. Afin de disposer d'un tel outil, nous associons, à chaque valeur $q \geq 0,2$ valeurs symboliques $q^{+}$et $q^{-}$, et étendons à ces valeurs symboliques la fonction Coût Généralisé $C_{f}$ définie plus haut, en posant :

$C_{f}\left(q^{+}, e\right)=$ limite de $C_{f}(q+\delta)$, pour $\delta->0^{+} ; C_{f}\left(q^{-}, e\right)=$ limite de $C_{f}(q+\delta)$, pour $\delta->0^{-}$.

Nous obtenons alors :

Théorème 3. Si un Circuit Généralisé Négatif $(\Gamma, q)$ existe pour le réseau $G^{*}{ }_{f}$ et pour la fonction $C_{f}$, alors $q$ peut être pris dans l'Ensemble Significatif $T_{f}=\left\{t^{+}, t^{-}\right.$ pour $\left.t \in[0,1] \cap\left(\{0,1\} \cup\left\{s\left(f, e^{*}\right), t\left(f, e^{*}\right), e^{*} \in E^{*}\right\}\right)\right\}$.

Démonstration. Si $\Gamma$ est un circuit de $G *_{f}$ et si $q>0, t$ et $t^{\prime} \in\{0,1\} \cup\left\{s\left(f, e^{*}\right)\right.$, $\left.t\left(f, e^{*}\right), e^{*} \in E^{*}\right\}$, sont tels que $t<q<t^{\prime}$, alors $L_{f}(\Gamma, q)$ dépend linéairement de $q$ entre $t$ et $t^{\prime}$. Si donc $L_{f}(\Gamma, q)<0$, alors l'une des valeurs $L_{f}\left(G, t^{+}\right), L_{f}\left(G, t^{\prime}\right)$ est négative. Si par ailleurs $q$ est strictement plus grand que 1 , alors $L_{f}(\Gamma, q)=$ $L_{f}(\Gamma, 1)+L_{f}(\Gamma, q-1)$. Le résultat s'en déduit.

De ce résultat, nous pouvons déduire une procédure de recherche d'un circuit généralisé négatif, nommée CYCLE-SEARCH, qui parcourt $T_{f}$ par valeurs croissantes et cherche, pour chaque valeur $q$ dans $T_{f}$, un circuit négatif de $G^{*}$ pour les coûts $C_{f}\left(q, e^{*}\right), e^{*} \in E^{*}$. Cet algorithme, qui n'est qu'heuristique, du fait de la Contrainte de Longueur, est une adaptation de l'algorithme des plus courts chemins de Ford. Nous faisons en sorte que $G *=\left(X, E^{*}\right)$ soit fortement connexe, considérons un sommet de référence $x_{o}$, et faisons, pour $q$ donné dans $T_{f}$, évoluer une antiarborescence $\Lambda$ de racine $x_{o}$, et couvrant les sommets de $G$, comme suit :

\section{Procédure CYCLE-SEARCH}

$f$ est donné, compatible avec les capacités $C \min$ et $C \max$, ainsi qu'une antiarborescence courante $\Lambda$ de racine $x_{o}$ dans le réseau $G^{*}{ }_{f}$;

Construire l'Ensemble Significatif $T_{f}$ associé à $f ; q=0+$; Not Stop;

Tant que (Not Stop) et $(q \leq 1)$ faire

Calculer les coûts $C_{f}(q, e), e \in E^{*}$;

Pour $x$ dans $X$, Calculer, au sens des coûts $C_{f}(q, e), e \in E^{*}$, la distance $D_{f}(q, x)$ de $x$ à $x_{o}$ dans $\Lambda$; 


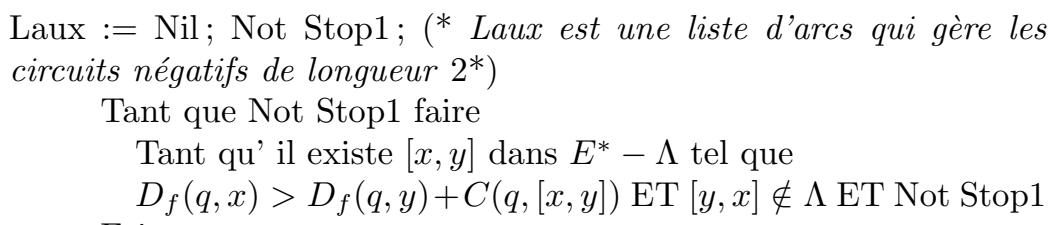
Faire

Si (remplacer dans $\Lambda$ l'arc sortant de $x$ par $[x, y]$ crée un circuit négatif $\left.\Gamma_{q}\right)$

Alors Stop1; Construire le cycle $\gamma_{q}$ associé à $\Gamma_{q}$ dans $G$; Sinon

Stop ; $(*)$

Retirer de $\Lambda$ l'arc sortant de $x$ et Insérer l'arc $[x, y]$ dans $\Lambda$;

Recalculer les quantités $D_{f}(q, x), x \in X$;

Si Not Stop1 ET (il existe $[y, x]$ dans $\Lambda$ - Laux et $[x, y]$ dans $E^{*}-\Lambda$ formant un circuit négatif)

Alors

Insérer $[y, x]$ dans Laux; Soit $e$ l'arc sortant de $x$ dans $\Lambda$; Choisir un arc $[y, z]$ de poids $C_{f}$ minimal et tel que $\Lambda$ $\{e,[y, x]\}+\{[x, y],[y, z]\}$ reste une antiarborescence Sinon Stop1;

Si Stop alors le résultat est fourni par l'instruction $(*)$ si Stop, et sinon Echec;

Commentaire : le rôle de la liste auxiliaire Laux est ici de garantir la terminaison de l'algorithme, et d'éviter l'occurence d'une boucle infinie de génération des circuits négatifs de longueur 2.

\section{Un algorithme pour le problème FCEM}

Nous déduisons de ce qui précède un algorithme simple et naturel, de nature heuristique, pour le problème FCEM, que nous nommons CYGEN (Cycles Généralisés) :

Algorithme CYGEN (Résolution du problème FCEM)

Initialiser $f$, compatible avec les capacités $C$ min et $C \max$; Not Stop ;

Tant que Not Stop faire

Soit $\Lambda$ une antiarborescence de racine $x_{o}$ dans le réseau $G_{f}^{*}$; Not Stop ;

Appliquer la procédure CYCLE-SEARCH à $f$ et $\Lambda$;

Si CYCLE-SEARCH produit un cycle généralisé améliorant $\gamma, q$ alors $f:=f+q \cdot \phi_{\gamma}$

Sinon Stop (le flot courant $f$ devient le résultat fourni par le processus).

4.2. Un MÉCANISME D'AGRÉGATION/DÉSAGRÉGATION POUR LE PROBLÈME CFEMF-OD

Comme il a été dit tant en section 1 qu'au début de la section 4, nous voulons pouvoir, dans le cas ou $K$ est grand, traiter $\mathbf{P}$-aux en appliquant directement la 
procédure CYGEN ci-dessus au flot agrégé $\operatorname{Sum}(f)$. En effet, dans le cas où le multiflot $f$ comporte un grand nombre de composants flots, dont les valeurs sont petites par rapport à l'unité, traiter séparément chaque flot $f(k)$ tend rapidement à ne plus altérer $\lceil\operatorname{Sum}(f)\rceil$, c'est-à-dire à ne plus créer de regroupement autour de la ressource associée à $F$. Le but de cette section est donc d'appliquer directement CYGEN au flot agrégé Sum $(f)$, de telle sorte que celui-ci reste décomposable comme somme des composants $f(k), k \in K$, d'un multiflot $f$ compatible avec les capacités $C$ min et $C$ max.

Pour plus de simplicité, nous nous limitons au cas particulier du problème CFEMF-OD, qui est assez représentatif des situations que nous cherchons à gérer à l'aide de d'un tel mécanisme d'agrégation :

Problème CFEMF-OD :

$\{$ Input : le réseau $G=(X, E)$, un sous-ensemble $A$ de $E$, des vecteurs coûts $c \geq 0$ et $p \geq 0$, indexés sur les arcs de $G$, une famille $O D=\left\{\left(o_{k}, d_{k}\right), k \in\right.$ $K\}$ de couples de sommets origine/destination, et une famille $D=\left\{D_{k}\right.$, $k$ dans $K\}$ de demandes associées.

Objectif : trouver un flot $F \geq 0$, entier, et un multiflot $f=\{f(k), k \in$ $K\} \geq 0$, tels que:

- pour tout $k$ dans $K, f(k)$ achemine la quantité $D_{k}$ de $o_{k}$, vers $d_{k}, ;(f$ achemine $D$ vers $O D$ ),

$-F_{A} \geq \operatorname{Sum}(f)_{A}$

et qui minimisent la quantité c.F + p.Sum $(f)\}$.

Nous disons alors qu'un flot $g \geq 0$ défini sur $\mathrm{G}$ est décomposable par rapport au couple $(O D, D)$, s'il existe un multiflot $f=\{f(k), k$ dans $K\} \geq 0$, qui soit $d$ 'Acheminement de $D$ vers $O D$, et tel que $\operatorname{Sum}(f)=g$. Il est dit chemin-décomposable s'il est possible de faire en sorte que chaque flot $f(k)$ soit un multiple d'un flot chemin.

Le problème CFEMF-OD se réécrit donc sous la forme :

trouver un flot $F \geq 0$, entier, et un flot $g$ décomposable par rapport à $D$ et $O D$, tel que $F_{A} \geq g_{A}$,

et qui minimisent la quantité c.F + p.g\},

et le problème auxiliaire $\mathbf{P}$-aux, qui lui est associé selon la section 3, prend la forme FACEM (Flot Agrégé de Coût Entier Minimal) suivante :

$\left\{\right.$ Input : $\lambda \geq 0$ est un vecteur coût indexé sur $E$ et tel que $\lambda_{A}=0$.

Objectif : trouver un flot $g$ décomposable par rapport à $D$ et $O D$, et minimisant $\lambda .\lceil g\rceil+p . g\}$.

Nous simplifions ces deux problèmes en faisant l'Hypothèse de Non Bifurcation, c'est-à-dire en imposant que le flot $g$ soit chemin-décomposable par rapport à $D$ et $O D$. Cette hypothèse, nous permettra de gérer la contrainte de décomposabilité à l'aide de procédures simples de recherche de chemins dans des graphes. Testée en [9], elle s'avère peu restrictive quand $O D$ est assez grand, ce que nous pouvons 
confirmer grâce au résultat suivant :

Théorème 4. Supposons $G$ fortement connexe. On peut alors trouver une solution optimale $\left(F^{*}, f^{*}\right)$ de $\boldsymbol{C F E M F - O D}$, telle que au plus $\operatorname{Card}(E)-\operatorname{Card}(X)+1$ composants de $f$ ne soient pas des flot-chemins.

Démonstration. Considérons une solution optimale $\left(F^{*}, f^{*}\right)$ du problème CFEMF-OD, et supposons que $N$ composants de $f^{*}$, numérotés $f^{*}(1) \ldots f^{*}(N)$, ne sont pas des flots-chemins, avec $N>\operatorname{Card}(E)-\operatorname{Card}(X)+1$. Supposons aussi que $F^{*}$ et $f^{*}$ ont été choisis de telle sorte que $N$ soit minimal et que pour cette valeur $N$, la somme $U=\Sigma_{n=1 \ldots N} U_{n}$ soit minimale, $U_{n}$ étant le nombre d'arcs $e$ de $G$ tels que $f(n)_{e} \# 0$. Il existe dès lors, pour chaque $i=1 \ldots N$, un cycle élémentaire $\gamma_{n}$ formé d'arcs dans $U_{n}$, et les flots-cycles $\phi_{\gamma n}, n=1 \ldots N$, qui sont associés à ces cycles sont linéairement dépendants. Supposons qu'on puisse écrire $\Sigma_{=1 \ldots N} t_{n} . \phi_{\gamma n}=0$, où les coefficients $t_{n}$ sont non tous nuls, et considérons un petit nombre $\lambda \geq 0$. On peut remplacer, pour tout $n=1 \ldots N, f(n) \operatorname{par} f(n)+\lambda . t_{n} . \phi_{\gamma n}$. Ce faisant, on ne change $\operatorname{Sum}(f)$. Il suffit alors de choisir $\lambda$ le plus grand possible et tel que chaque flot $f(n)$ demeure $\geq 0$, pour déduire une contradiction sur la minimalité de $N$ et de la quantité $U$.

Cette contrainte de Décomposabilité est malheureusement difficile à gérer. Nous pouvons observer qu'une condition nécessaire de décomposabilité est fournie par la famille de contraintes dites Inégalités de Coupes, qui constituent un cas particulier des Coupes Métriques (voir [9,21,26]), et qui se formulent ainsi :

pour toute partie de $Z$ de $X, \Sigma_{e \text { sort de } Z} g_{e} \geq \Sigma_{k \text { dans } O D(Z)} D_{k}$, où $O D(Z)=\{k \in$ $K$ tels que $o_{k} \in Z$ et $\left.d_{k} \in X-Z\right\}$.

Cette remarque nous conduit à un traitement itératif de FACEM, selon lequel à chaque étape :

- un cycle généralisé améliorant $(\gamma, q)$ est recherché (adaptation de la procédure CYCLE-SEARCH), pour le flot courant $g$, de telle sorte que le flot résultant $g+q . \phi_{\gamma q}$ ne viole aucune des contraintes présentes au sein d'un ensemble $\Delta$ d'inégalités de coupes courantes;

- en cas de succès, une décomposition de $g$ ainsi perturbé en flots-chemins compatibles avec $D$ et $O D$ est alors recherchée. En cas d'échec de cette dernière recherche, nous procédons à :

- la génération d'inégalités de coupes insatisfaites par $g$, qui sont lors injectées dans la famille $\Delta$;

- une correction de $g$ de façon à ce qu'il demeure chemin-décomposable par rapport $D$ et $O D$.

Ce traitement se synthétise comme suit :

Algorithme CYGEN-AGREG (traitement du problème FACEM).

$1^{\text {re }}$ phase : Initialisation de $g$;

Ordonner les éléments de $K$ par quantités $\mathrm{D}_{k}$ décroissantes; $g:=0 ; \Delta:=$ Nil ; 
Pour $k$ dans $K$ ainsi ordonné faire :

Calculer un plus court $\left(o_{k}-d_{k}\right)$-chemin $\Gamma_{k}$ dans $G$, pour les coûts $\alpha_{e}=D_{k} \cdot p_{e}+\lambda_{e} \cdot\left(\left\lceil g_{e}+D_{k}\right\rceil-\left\lceil g_{e}\right\rceil\right), e \in E$;

Ajouter à $g$ le flot réalisant le transport de $D_{k}$ depuis $o_{k}$ vers $d_{k}$ le long de $\Gamma_{k}$;

Ajouter à $\Delta$ les coupes associées à la construction de $\Gamma_{k}$ selon l'algorithme de Dijskstra;

Poser $\Sigma:=\left\{\Gamma_{k}, k \in K\right\}$;

$2^{e}$ phase : Amélioration de $g$ par itérations successives; (* SEUIL est ici un paramètre de contrôle*)

Not Stop; Compteur :=0;

Tant que Not Stop faire :

soit $\Lambda$ une antiarborescence de racine $x_{o}$ dans le réseau $G_{g}^{*}$ associé à $g$;

appliquer la procédure CYCLE-SEARCH à $f$ et $\Lambda$, en l'adaptant de façon à ne générer que des cycles généralisés $(\gamma, q)$ tels que $g+\phi_{\gamma}$ satisfait les coupes de $\Delta$;

si CYCLE-SEARCH produit un tel cycle généralisé améliorant $\gamma, q$ alors

perturbation de $g$ et incrémentation de $\Delta$ (génération de coupes) :

$h:=g+q . \phi_{\gamma} ;$ Not Stop1;

Tant que Not Stop1 ET $g<>h$ faire

$\delta:=\operatorname{Sup}_{e \in E}\left\lceil(g-h)_{e}\right\rceil$;

Chercher $k \in K$ et un chemin $\Gamma$ de $o_{k}$ vers $d_{k}$, tels que remplacer $\Gamma_{k}$ par $\Gamma$ dans la définition de $g$ induit une diminution stricte de la quantité Sup $e_{e \in E}\left\lceil(g-h)_{e}\right\rceil$;

Si $k$ et $\Gamma$ existent alors remplacer $\Gamma_{k}$ par $\Gamma$ dans $\Sigma$ et modifier $g$ en conséquence

Sinon

Stop1; Déduire, pour chaque $k \in K$, une partition $Z_{k}$, $X-Z_{k}$ de $X$ associée à l'échec de la construction de $\Gamma$, (recherche de chemin dans un graphe orienté), et intégrer dans $\Delta$ l'inégalité de coupe associée à $Z_{k}$;

Si la quantité $\lambda .\lceil g\rceil+p . g$ n'a pas diminué alors

Compteur := Compteur +1 ; Si Compteur > SEUIL alors Stop;

Sinon Stop (le flot courant $g$ est le résultat fourni par le processus);

\subsection{Traitementde l'Instruction (I1) du Schéma DME de la section 3}

Cette instruction commande, pour un multiflot courant $f$ et un vecteur dual $\lambda$, l'exécution d'une itération d'un processus de résolution du problème $\mathbf{P}$-aux, et ce en vue de diminuer la valeur de la quantité $\lambda$. $\lceil\operatorname{Sum}(f)\rceil+p . \operatorname{Sum}(f)\}$. Dans le cas 
du Problème CFEM-OD, nous pouvons la réaliser en procédant comme suit :

Instruction (I1), Schéma DME, Problème CFEM-OD : appliquer, à partir de $g=\operatorname{Sum}(f)$ courant, une itération de la boucle principale de la $2^{\mathrm{e}}$ phase de la procédure CYGEN-AGREG.

\section{Tests NumÉRIQUeS}

Nous présentons ici quatre classes de tests, effectués sur un IBM Power PC 604o, 100 Mhz, 1 Go mémoire vive, à l'aide de la bibliothèque CPLEX (version déjà ancienne 6.0, Callable Library utilisée depuis un compilateur C, gcc 2.95.2) :

- la première évalue les schémas de résolution DRCOUP, DRFLOT et DME présentés en section 3 ;

- la deuxième vise à analyser le comportement de l'algorithme CYGEN de la section 4.1 ;

- la troisième concerne les performances de l'algorithme CYGEN-AGREG de la section 4.2 ;

- la quatrième vise à permettre l'analyse du comportement de la procédure heuristique DME-CYGEN, qui constitue l'heuristique résultant de l'intégration, au niveau de l'instruction (I1) du schéma DME de la section 3.1, des méthodes présentées en section 4.

Nous utiliserons d'une façon générale des graphes proches de ceux que l'on peut rencontrer dans les systèmes de transports, c'est-à-dire faiblement denses (faible rapport $\operatorname{Card}(E) / \operatorname{Card}(X))$, cette dernière caractéristique ne semblant avoir que peu d'impact sur le comportement des algorithmes, d'abord déterminés par les nombres d'arcs, de composants du multiflot, et les caractéristiques des vecteurs coûts et demandes.

\section{1. $1^{\text {re }}$ catégorie de tests : COMPARAISON DES SCHÉMAS DRCOUP, DRFLOT ET DME}

Cette première expérimentation évalue donc les schémas DRCOUP, DRFLOT et DME présentés en section 3 et à les comparer. Elle ne concerne que le cas où le multiflot $f$ est en fait un flot $(\operatorname{Card}(K)=1)$. Les schémas DRCOUP et DRFLOT sont entièrement traités selon une séquence d'appels de la bibliothèque CPLEX sur les programmes mixtes induits par les relaxations lagrangiennes associées. Le schéma DME est implémenté sous la forme proposée en section 3.1, l'instruction portant sur la perturbation du multiflot $f$ étant alors traitée à l'aide de l'algorithme CYGEN de la section 4 (schéma DME-CYGEN), et les appels à CPLEX ne concernant que la résolution du sous-problème relatif au flot entier $F$.

Elle vise d'abord à comparer entre elles les valeurs produites par ces schémas de résolution. Elle est réalisée sur des instances du problème CFEMF de petites tailles, c'est-à-dire n'induisant pas la présence de plus d'une centaine de variables entières, de façon à permettre l'obtention de résultats exacts par CPLEX, 
Tableau 1. Descriptif des tests.

\begin{tabular}{|l|l|l|l|l|l|l|}
\hline Test & $C X$ & $C E$ & $C A$ & $C c$ & $C p A$ & $C p A^{*}$ \\
\hline 1 & 10 & 30 & 10 & 0,5 & 0,4 & 1,2 \\
\hline 2 & 10 & 30 & 10 & 1 & 0,2 & 1,4 \\
\hline 3 & 10 & 30 & 10 & 2 & 0,2 & 1,6 \\
\hline 4 & 20 & 60 & 15 & 0,5 & 0,4 & 1,2 \\
\hline 5 & 20 & 60 & 15 & 1 & 0,2 & 1,4 \\
\hline 6 & 20 & 60 & 15 & 2 & 0,2 & 1,6 \\
\hline 7 & 15 & 50 & 25 & 0,5 & 0,4 & 1,2 \\
\hline 8 & 15 & 50 & 25 & 1 & 0,2 & 1,4 \\
\hline 9 & 15 & 50 & 25 & 2 & 0,2 & 1,6 \\
\hline
\end{tabular}

et présentant les caractéristiques suivantes : (rappelons que le flot entier $F$ est indexé sur l'ensemble $E$ des arcs de $G$ )

$C X=\operatorname{Card}(X) \in\{10 \ldots 20\} ; C E=\operatorname{Card}(E) \in\{30 \ldots 50\} ;$

$\operatorname{Card}(A) / \operatorname{Card}(E) \in[0,25,0,5] ;$ nous posons $C A=\operatorname{Card}(A)$;

$0 \leq C \min \leq 5 ; C \min \leq M A X \leq C \min +5$;

les composantes de $p$ et de $c$ sont générées entre 0 et $10: C c$ est la moyenne des coûts de $c, C p A$ est la moyenne des coûts de $p$ sur les $\operatorname{arcs}$ de $A$, et $C p A^{*}$ est la moyenne des coûts de $p$ sur les arcs hors de $A$.

Les instances considérées n'ont pas de sémantique particulière.

Les quantités que nous cherchons alors à observer sont, pour chaque test :

$V^{*}=$ valeur optimale de la relaxation fractionnaire du problème CFEMF considéré;

$V=$ valeur optimale du problème CFEMF considéré;

$V$-DME $=$ valeur produite par le schéma DME-CYGEN

$V$-COUP $=$ valeur produite par le schéma DRCOUP ;

$B=$ valeur produite par la phase 1 du schéma DRCOUP ;

$V$-FLOT $=$ valeur produite par le schéma DRFLOT;

$D=$ Valeur produite par la phase 1 du schéma DRFLOT.

Nous ne fournissons pas non plus ici de temps CPU, dans la mesure où les valeurs associées aux schémas DRCOUP et DRFLOT n'ont pas fait l'objet d'un travail algorithmique particulier. Les autres expérimentations, qui auront pour objet de mieux tester les algorithmes présentés en section 4, contiendront ce type d'information.

Nous obtenons : (rappel : le nombre $K$ de composants du multiflot $f$ est ici égal à 1).

Commentaire : nous constatons que les valeurs $B, D$ et $V$-DME sont ici en général très proches de $V$, ce qui signifie que les différents schémas DRFLOT, DRCOUP et DME constituent de bonnes approximations pour le traitement des problèmes CFEMF considérés : erreurs moyennes respectivement égales à $3 \%$ et $4 \%$. Ceci est à pondérer toutefois avec le fait que, dans le cadre des instances traitées ici, la relaxation de la contrainte d'intégrité a fourni elle aussi des résultats 
TABleau 2. Résultats associés.

\begin{tabular}{|l|l|l|l|l|l|l|l|}
\hline Test & $V^{*}$ & $V$ & $V$-DME & $V$-COUP & $B$ & $V$-FLOT & $D$ \\
\hline 1 & 57,5 & 61,2 & 61,8 & 64,2 & 60,5 & 66,7 & 59,9 \\
\hline 2 & 59,1 & 65,5 & 68,7 & 74,0 & 63,2 & 71,6 & 63,1 \\
\hline 3 & 66,1 & 72,3 & 75,4 & 78,7 & 70,5 & 75,6 & 71,5 \\
\hline 4 & 105,8 & 125,0 & 127,2 & 127,3 & 124,6 & 133,7 & 124,5 \\
\hline 5 & 117,2 & 134,7 & 148,6 & 152,1 & 125,5 & 158,3 & 129,6 \\
\hline 6 & 135,9 & 151,6 & 154,0 & 154,0 & 149,8 & 168,7 & 149,4 \\
\hline 7 & 105,4 & 121,3 & 123,6 & 129,7 & 119,8 & 126,0 & 119,1 \\
\hline 8 & 112,2 & 132,6 & 144,4 & 147,3 & 125,0 & 147,6 & 125,0 \\
\hline 9 & 121,9 & 145,8 & 147,9 & 151,8 & 141,4 & 155,2 & 141,4 \\
\hline
\end{tabular}

relativement proches des optimae (erreur moyenne de $13 \%$ ). Les phases de projection associées à DRFLOT et DRCOUP fournissent des résultats contrastés (erreurs moyennes respectives de $7 \%$ et $9 \%$ ), sensiblement moins bons que ceux fournis par DME-CYGEN, ce qui explique que pour les tests de la section 5.4, qui concernent des problèmes de type CFEM-MOD dans lequel l'objet $f$ est un véritable multiflot, nous nous soyons limités à l'étude du schéma DME-CYGEN.

Les valeurs de $C c, C p A$ et $C p A^{*}$ n'influent par ailleurs que peu sur les écarts entre les valeurs $B, D$ et $V$-DME. Elles influent par contre sur la structure des solutions et sur les coûts respectifs des flots $F$ et $f$. En dehors des plages induites par ces tableaux pour les coefficients $C c, C p A$ et $C p A^{*}$, on constate qu'il y a soit recours systématique pour le flot $f$ aux arcs de $A$ (dans le cas ou le coût moyen $C c$ pèse sensiblement moins que la différence $\left.C p A^{*}-C p A\right)$, soit au contraire, évitement systématique des arcs de $A$ par le flot $f$ (dans le cas ou $C c$ domine sensiblement $C p A^{*}-C p A$ ), ce qui induit alors que $F$ n'existe quasiment pas. De fait, ces plages correspondent aux valeurs des paramètres $c$ et $p$ qui font que le problème présente vraiment une difficulté.

\section{2. $2^{\text {e }}$ CATÉgorie DE TESTS : L'Algorithme CYGEN ET LE PROBLÈME FCEM}

Cette catégorie de tests concerne le problème FCEM défini en section 4.1. Les réseaux considérés sont générés aléatoirement de façon à présenter les caractéristiques suivantes :

$C X=\operatorname{Card}(X) \in\{20,60\}$

$C E=\operatorname{Card}(E) \in\{60,400\}$;

$\operatorname{Card}(\mathrm{A}) / \operatorname{Card}(\mathrm{E}) \in[1 / 4,1 / 2] ;$ nous posons $C A=\operatorname{Card}(A) ;(\operatorname{rappelons}$ que le vecteur $\lambda$ est nul sur $E-A)$;

les composantes de p et $\lambda$ sont générées entre 0 et $5: C \lambda$ est la moyenne des coûts de $\lambda, C p A$ est la moyenne des coûts de $p$ sur les arcs de $A$ et $C p A^{*}$ est la moyenne des coûts de $p$ sur les arcs hors de $A$.

CYGEN considère l'ensemble significatif $T_{f}$, comme étant l'ensemble des multiples de 0,05, ce qui nous permet d'en limiter la taille, et ne semble pas avoir d'impact excessif sur le fonctionnement de l'algorithme. 
Tableau 3. Descriptif des tests.

\begin{tabular}{|l|l|l|l|l|l|l|}
\hline Test & $C X$ & $C E$ & $C A$ & $C \lambda$ & $C p A$ & $C p A^{*}$ \\
\hline 1 & 20 & 60 & 30 & 0,5 & 0,4 & 1,2 \\
\hline 2 & 20 & 60 & 30 & 1 & 0,2 & 1,4 \\
\hline 3 & 20 & 60 & 30 & 2 & 0,2 & 1,6 \\
\hline 4 & 40 & 150 & 50 & 0,5 & 0,4 & 1,2 \\
\hline 5 & 40 & 150 & 50 & 1 & 0,2 & 1,4 \\
\hline 6 & 40 & 150 & 50 & 2 & 0,2 & 1,6 \\
\hline 7 & 60 & 400 & 100 & 0,5 & 0,4 & 1,2 \\
\hline 8 & 60 & 400 & 100 & 1 & 0,2 & 1,4 \\
\hline 9 & 60 & 400 & 100 & 2 & 0,2 & 1,6 \\
\hline
\end{tabular}

TABleau 4. Résultats associés.

\begin{tabular}{|l|l|l|l|l|l|l|l|}
\hline Test & $R E$ & $T-R E$ & $O P T$ & $T-O P T$ & $V$-CY & T-CY & ITER-2 \\
\hline 1 & 60,6 & 0,1 & 68,2 & 13 & 68,2 & 1 & 3,8 \\
\hline 2 & 80,3 & 0,2 & 86,7 & 98 & 88,0 & 5 & 6,7 \\
\hline 3 & 89,4 & 0,1 & 95,3 & 48 & 96,3 & 4 & 4,3 \\
\hline 4 & 175,0 & 0,5 & 190,2 & 92 & 194,6 & 9 & 3,4 \\
\hline 5 & 208,7 & 1 & 222,7 & 941 & 224,8 & 48 & 5,0 \\
\hline 6 & 237,8 & 0,8 & 246,3 & 680 & 256,3 & 31 & 6,7 \\
\hline 7 & 358,3 & 2 & 404,8 & 851 & 404,8 & 76 & 3,2 \\
\hline 8 & 461,7 & 7 & 492,0 & 4874 & 500,6 & 147 & 5,2 \\
\hline 9 & 524,6 & 4 & 549,6 & 3304 & 576,3 & 120 & 4,0 \\
\hline
\end{tabular}

Les quantités que nous cherchons alors à observer sont, pour chaque test :

$R E=$ valeur optimale de la relaxation fractionnaire du problème FCEM (calculé avec la bibliothèque CPLEX);

$T-R E=$ temps $\mathrm{CPU}$ associé;

$O P T=$ valeur optimale du problème FCEM (calculée directement avec la bibliothèque CPLEX) $=1$;

$T-O P T=$ temps CPU associé;

$V-C Y=$ valeur trouvée par l'algorithme CYGEN;

$T-C Y=$ temps CPU associé;

ITER-2 : nombre moyen de valeurs $t$ dans $T_{f}$ testées à chaque itération de la boucle principale du programme CYGEN (nombre d'itérations de la boucle interne).

Nous obtenons les tableaux 3 et 4 (rappelons que le nombre de variables entières dans le programme linéaire associé au problème traité FCEM est fourni ici par $C A$ ).

Commentaires : l'algorithme CYGEN fournit ici en général des résultats très proches de l'optimum (erreur moyenne autour de $2 \%$ ). Les temps relativement longs pour l'exécution de CYGEN sont dûs au fait que son implémentation n'est 
pas optimisée. Par ailleurs, les remarques faites à l'issue de la section précédente à propos du rôle joué par les coefficients $C \lambda, C p A$ et $C p A^{*}$ valent sensiblement de la même façon ici. Deux tests seulement sur les neuf présentés ici voient CYGEN fournir le résultat optimum. Cette proportion n'est pas très élevée, ce qui s'explique d'une part par le fait que l'absence d'un cycle généralisé améliorant n'est qu'une condition nécessaire d'optimalité pour le problème que nous traitons ici, et d'autre part par le fait que le procédé de recherche d'un tel cycle que nous utilisons ici n'est qu'heuristique. Nous n'avons pu distinguer la part qui revient ici à chacune de ces deux causes possibles de non optimalité, car il nous aurait fallu pouvoir nous appuyer, afin de tester ce point, sur une procédure exacte de recherche de cycle généralisé améliorant, ce dont nous ne disposons pas.

\section{3. $3^{\text {e }}$ CATÉGorie de tests : L'Algorithme CYGEN-AGREG ET LE PROBLÈME FACEM}

Cette catégorie de tests concerne le problème FACEM défini en section 4.2. Les réseaux considérés satisfont :

$$
\begin{aligned}
& C X=\operatorname{Card}(X) \in\{10,60\} ; \\
& C E=\operatorname{Card}(E) \in\{30,400\} ; \\
& K=\operatorname{Card}(O D) \in\{10,200\} ;
\end{aligned}
$$

$\operatorname{Card}(A) / \operatorname{Card}(E) \in[1 / 4,1 / 2] ;$ nous posons $C A=\operatorname{Card}(A) ;(\operatorname{rappelons}$ que le vecteur $\lambda$ est nul sur $E-A)$;

les composantes de $p$ et $\lambda$ sont générées entre 0 et $5: C \lambda$ est la moyenne des coûts de $\lambda, C p A$ est la moyenne des coûts de $p$ sur les $\operatorname{arcs}$ de $A$ et $C p A^{*}$ est la moyenne des coûts de $p$ sur les arcs hors de $A$.

Les composantes de $D$ sont générées entre 0 et 1 : nous notons $C D$ la moyenne des coefficients $D_{k}, k \in K$.

Les quantités que nous cherchons alors à observer sont, pour chaque test :

$R E=$ valeur optimale de la relaxation fractionnaire du problème FACEM

(calculée avec CPLEX);

$T-R E=$ temps CPU associé;

$O P T=$ valeur optimale du problème FACEM (calculée avec la bibliothèque CPLEX);

$T-O P T=$ temps CPU associé;

$V-A C Y=$ valeur trouvée par l'algorithme CYGEN-AGREG;

$T-A C Y=$ temps CPU associé;

$N-C O=$ nombres de coupes métriques gérées par le processus ;

$N A=$ nombre d'arcs e de $A$ portant des valeurs $F_{e}$ non nulles selon CYGEN-AGREG.

Nous obtenons les tableaux 5 et 6 (le nombre de variables entières dans le programme linéaire associé à FACEM est égal à $C A$ ).

Commentaire : l'erreur induite par CYGEN-AGREG se situe ici autour de $5 \%$. La gestion de l'agrégation n'entraine pas ici un surplus d'erreur considérable par rapport au cas testé en section antérieure 5.3. Les temps d'exécution associés 
Tableau 5. Descriptif des tests.

\begin{tabular}{|l|l|l|l|l|l|l|l|l|}
\hline Test & $C X$ & $C E$ & $C A$ & $C \lambda$ & $C p A$ & $C p A^{*}$ & $K$ & $C D$ \\
\hline 1 & 20 & 60 & 30 & 0,5 & 0,4 & 1,2 & 10 & 0,5 \\
\hline 2 & 20 & 60 & 30 & 1 & 0,2 & 1,4 & 10 & 0,5 \\
\hline 3 & 20 & 60 & 30 & 2 & 0,2 & 1,6 & 10 & 0,5 \\
\hline 4 & 40 & 150 & 50 & 0,5 & 0,4 & 1,2 & 50 & 0,2 \\
\hline 5 & 40 & 150 & 50 & 1 & 0,2 & 1,4 & 50 & 0,2 \\
\hline 6 & 40 & 150 & 50 & 2 & 0,2 & 1,6 & 50 & 0,2 \\
\hline 7 & 60 & 400 & 100 & 0,5 & 0,4 & 1,2 & 200 & 0,05 \\
\hline 8 & 60 & 400 & 100 & 1 & 0,2 & 1,4 & 200 & 0,05 \\
\hline 9 & 60 & 400 & 100 & 2 & 0,2 & 1,6 & 200 & 0,05 \\
\hline
\end{tabular}

TABLEAU 6. Résultats associés.

\begin{tabular}{|l|l|l|l|l|l|l|l|l|}
\hline Test & $R E$ & $T-R E$ & $O P T$ & $T-O P T$ & $V-A C Y$ & $T-A C Y$ & $N C O$ & $N A$ \\
\hline 1 & 48,6 & 2 & 57,5 & 17 & 58,6 & 14 & 39 & 22 \\
\hline 2 & 51,7 & 3 & 66,3 & 140 & 71,3 & 21 & 102 & 20 \\
\hline 3 & 54,4 & 2 & 70,4 & 108 & 72,8 & 22 & 108 & 14 \\
\hline 4 & 132,5 & 8 & 185,8 & 165 & 188,0 & 73 & 252 & 25 \\
\hline 5 & 144,0 & 12 & 204,7 & 2408 & 220,6 & 165 & 288 & 24 \\
\hline 6 & 149,3 & 11 & 210,9 & 927 & 222,9 & 102 & 267 & 17 \\
\hline 7 & 267,2 & 64 & 396,0 & 2825 & 402,6 & 309 & 431 & 37 \\
\hline 8 & 294,8 & 108 & Echec & Echec & 482,4 & 1255 & 602 & 34 \\
\hline 9 & 306,9 & 99 & 455,7 & 9762 & 485,3 & 947 & 686 & 23 \\
\hline
\end{tabular}

à ces tests sont assez importants, partie car nos algorithmes n'ont pas été optimisés. On remarque que, pour aucun des tests présentés ici, la valeur fournie par CYGEN-AGREG ne coincide avec l'optimum. Cet algorithme fonctionne en effet de façon heuristique à la fois dans sa gestion du flot agrégé (algorithme CYGEN), et dans sa gestion du processus d'agrégation/désagrégation. Les paramètres de coût $\left(\mathrm{C} \lambda, C p A, C p A^{*}\right)$ et de demande $(K, C D)$, exercent enfin ici une double influence :

- le traitement des instances est en général plus coûteux en temps et moins précis quand les valeurs $C D$ sont faibles et quand les coûts $C \lambda$ équilibrent le différentiel $C p A-C p A^{*}$;

- le nombre d'arcs de $A$ portant des valeurs de flots non nulles décroit sensiblement au fur et à mesure que le coût $C \lambda$ croit, et le ratio $N A / C A$ décroit en même temps que croit $C A / C E$, c'est-à-dire en même temps que la part de $G$ qui est occupée par les arcs de $A$. 


\section{4. $4^{\text {e }}$ CATÉGORIE DE TESTS : L'AlGORIThME DME-CYGEN ET LE PROBlèmE CFEMF-OD}

Cette catégorie de tests concerne le problème CFEM-OD. Les réseaux considérés tels que :

$C X=\operatorname{Card}(X) \in\{10 \ldots 60\} ; \operatorname{Card}(\mathrm{E}) \in\{30 \ldots 400\} ;$ nous posons $C E=$ $\operatorname{Card}(E)$;

$K=\operatorname{Card}(O D) \in\{10 \ldots 200\}$;

$\operatorname{Card}(A) / \operatorname{Card}(E) \in[1 / 4,2 / 3] ;$ nous posons $C A=\operatorname{Card}(A)$;

les composantes de $p$ et $c$ sont générées entre 0 et 5 : nous notons $C c$ la moyenne des coûts de $c$, par $C p A$ la moyenne des coûts de $p$ sur les arcs de $A$ et par $C p A^{*}$ la moyenne des coûts de $p$ sur les arcs hors de $A$.

Les composantes de $D$ sont générées entre 0 et 1 : nous notons $C D$ la moyenne des coefficients $D_{k}, k \in K$.

Est principalement testée la procédure heuristique DME-CYGEN, qui constitue l'heuristique résultant de l'intégration, au niveau de l'instruction (I1) du schéma DME de la section 3.1, des méthodes CYGEN et CYGEN-AGREG présentées en section 4. Cette procédure appelle CPLEX au niveau de chaque résolution du sous-problème CFEMF-OD ${ }_{f}$, qui porte sur le flot entier $F$, le multiflot $f$ étant fixé.

Les quantités que nous cherchons alors à observer sont, pour chaque test :

$R E=$ valeur optimale de la relaxation fractionnaire du problème CFEMFOD (calculée avec la bibliothèque CPLEX);

$T-R E=$ temps CPU associé;

$O P T=$ valeur optimale du problème CFEMF-OD (calculée avec la bibliothèque CPLEX);

$T-O P T=$ temps CPU associé;

$V-A C Y=$ valeur trouvée par l'algorithme DME-CYGEN;

$T-A C Y=$ temps CPU associé;

$I T E R=$ nombre d'itétations de la boucle principale du schéma DME;

$N A=$ nombre d'arcs $e$ de $A$ tels que $F_{e}$ est non nul selon DME-CYGEN.

Nous obtenons les tableaux 7 et 8 (NB : le nombre de variables entières du programme linéaire associé est ici égal à $\mathrm{CE}$ )

Commentaires : le problème FCEMF-OD est structurellement assez proche du problème FACEM. Les temps d'éxécution de l'algorithme DME-CYGEN sur le problème CFEMF-OD sont sont d'un ordre de grandeur assez comparable, pour les mêmes instances, à ceux enregistrés pour l'algorithme CYGEN-AGREG sur le problème FACEM. Les deux algorithmes opèrent en effet sur les mêmes objets et le traitement, à chaque itération de la boucle principale de DME-CYGEN, de la restriction au flot entier $F$ problème CFEMF-OD, ne constitue pas une surcharge considérable. À l'opposé, les temps nécessaires à l'obtention d'une valeur exacte par CPLEX pour le problème CFEM-OD, augmentent très sensiblement, du fait bien sûr de la contrainte de flot sur $F$, mais aussi du fait que le flot entier $F$ est ici indexé sur l'ensemble des arcs du réseau $G$, ce qui augmente nettement le 
Tableau 7. Descriptif des tests.

\begin{tabular}{|l|l|l|l|l|l|l|l|l|}
\hline Test & $C X$ & $C E$ & $C A$ & $C c$ & $C p A$ & $C p A^{*}$ & $K$ & $C D$ \\
\hline 1 & 10 & 30 & 20 & 0,5 & 0,4 & 1,2 & 10 & 0,5 \\
\hline 2 & 10 & 30 & 20 & 1 & 0,2 & 1,4 & 10 & 0,5 \\
\hline 3 & 10 & 30 & 20 & 2 & 0,2 & 1,6 & 10 & 0,5 \\
\hline 4 & 20 & 60 & 30 & 0,5 & 0,4 & 1,2 & 20 & 0,3 \\
\hline 5 & 20 & 60 & 30 & 1 & 0,2 & 1,4 & 20 & 0,3 \\
\hline 6 & 20 & 60 & 30 & 2 & 0,2 & 1,6 & 20 & 0,3 \\
\hline 7 & 30 & 90 & 50 & 0,5 & 0,4 & 1,2 & 40 & 0,2 \\
\hline 8 & 30 & 90 & 50 & 1 & 0,2 & 1,4 & 40 & 0,2 \\
\hline 9 & 30 & 90 & 50 & 2 & 0,2 & 1,6 & 40 & 0,2 \\
\hline 10 & 60 & 400 & 100 & 0,5 & 0,4 & 1,2 & 200 & 0,05 \\
\hline 11 & 60 & 400 & 100 & 1 & 0,2 & 1,4 & 200 & 0,05 \\
\hline 12 & 60 & 400 & 100 & 2 & 0,2 & 1,6 & 200 & 0,05 \\
\hline
\end{tabular}

TABleAu 8. Résultats associés.

\begin{tabular}{|l|l|l|l|l|l|l|l|l|}
\hline Test & $R E$ & $T-R E$ & OPT & $T-O P T$ & $V$-ACY & $T$-ACY & ITER & NA \\
\hline 1 & 32,4 & 2 & 40,3 & 99 & 41,2 & 35 & 13 & 14 \\
\hline 2 & 38,6 & 7 & 48,1 & 282 & 52,7 & 92 & 38 & 13 \\
\hline 3 & 42,8 & 5 & 51,9 & 208 & 55,0 & 81 & 35 & 10 \\
\hline 4 & 62,5 & 18 & 79,4 & 1239 & 81,4 & 122 & 24 & 18 \\
\hline 5 & 71,7 & 29 & 92 & 3410 & 101,9 & 345 & 62 & 17 \\
\hline 6 & 75,9, & 19 & 97,8 & 1980 & 105,3 & 303 & 60 & 13 \\
\hline 7 & 95,3 & 38 & 117,5 & 9243 & 121,1 & 157 & 29 & 24 \\
\hline 8 & 113,8 & 257 & Echec & Echec & 143,6 & 906 & 94 & 21 \\
\hline 9 & 122,6 & 89 & 150,8 & 17525 & 159,2 & 437 & 48 & 14 \\
\hline 10 & 289,8 & 419 & Echec & Echec & 451,5 & 912 & 128 & 35 \\
\hline 11 & 355,2 & 2337 & Echec & Echec & 545,8 & 3588 & 249 & 27 \\
\hline 12 & 428,6 & 955 & Echec & Echec & 550,9 & 2854 & 301 & 19 \\
\hline
\end{tabular}

nombre de variables entières apparaissant dans la formulation PLNE du problème. L'erreur moyenne commise par DME-CYGEN se situe ici un peu au-delà de $6 \%$, soit un taux sensiblement un peu plus élevé que celui enregistré pour les tests de la section précédente 5.3, ainsi qu'à à celui enregistré en section 5.1 relativement au comportement du schéma DME. Les remarques faites en section 5.3 à propos du rôle joué par les coefficients $C c, C p A, C p A^{*}, C D$ et $K$ valent ici. On constate enfin une diminution du taux des arcs porteurs d'infrastructures et mesurés par 
$N A$, qui est due à l'augmentation des coûts associés au flot $F$, dès lors que celui-ci est susceptible de prendre des valeurs non nulles en dehors de $A$.

\subsection{5 e CATÉGORIE DE TESTS : UNE EXPÉRIMENTATION SUR UN PROBLÈME RÉEL : LE ROUTAGE DES CHARGES CONVOYÉES PAR UNE FLOTTE DE CAMIONS}

Nous avons enfin expérimenté nos algorithmes sur une version particulière du problème dit du «Less than Truck Load » (voir [49]), et en adaptant un modèle initialement conçu au département de Génie Industriel de l'Université d'Oklahoma, dans le cadre d'un partenariat avec un transporteur nord-américain, opérant dans les états du sud des USA.

Le projet à l'intérieur duquel s'insère cette modélisation est vaste et complexe. Pris dans sa globalité, il porte sur le pilotage d'une flotte de camions et de remorques, qui convoient, entre différentes plate-formes logistiques fonctionnant en tant qu'interfaces entre des boucles locales et un réseau central (backbone), des chargements assemblés et désassemblés au niveau de ces plate-formes. Une fois constitués, ces chargements sont faits de remorques entières ou de containers portés par ces remorques .

À intervalles réguliers (quelques heures), l'opérateur doit, en fonction des demandes en attente et des demandes prévisionnelles, constituer les assemblages de remorques et containers qui vont être raccrochés aux camions, et router ceux-ci entre les plate-formes. Les cheminements suivis peuvent transiter par des nœuds « Relais », sur lesquels les camions peuvent échanger tout ou partie de leurs chargements.

Tel quel, le problème ainsi résumé combine de l'affectation de charges, du routage, de l'ordonnancement, et de la prise en compte d'aléas et d'incertitudes sur les demandes. Il n'a fait l'objet que de peu d'études dans la littérature, chaque transporteur nord-américain disposant de ses propres outils logiciels «ad-hoc». Afin de simplifier le traitement de ce problème, et compte tenu du fait que $80 \%$ de l'activité du transporteur dans le type de produits considérés (approvisionnements industriels) est récurrente, il a été défini un modèle agrégé qui vise à spécifier, pour une période donnée, les parcours suivis par les flux transportés et la façon dont ces parcours sont «mutualisés $»$. Ce modèle fait l'impasse sur les horaires et sur les mécanismes de synchronisation auxquels ces parcours vont donner lieu. Les solutions issues de ce modèle agrégé sont par la suite utilisées comme entrées pour d'autres modules qui tendent à dimensionner l'ensemble de véhicules utilisés, et à planifier de façon effective les parcours ainsi envisagés, ainsi que les échanges auxquels ils donnent lieu.

C'est donc sur ce modèle agrégé, qui s'intègre dans notre formalisme flot/multiflot avec contraintes de capacités et contraintes d'intégrité, et sur les données induites par le contexte d'application, que nous avons testé nos méthodes. Ce modèle se présente comme suit :

$P$ désigne l'ensemble des plate-formes logistiques et $R$ l'ensemble des nœuds relais. On travaille de fait dès lors sur un réseau $G=(X, E)$, tel 
que $X=P \cup R$, et qui présente des caractéristiques proches de celles des réseaux symétriques planaires. Pour tout arc $e=[x, y]$ de $G$, on connaît :

- le coût $p_{e}$ de circulation d'un camion vide sur $e$ (sensiblement proportionnel au temps de parcours prévisible);

- le coût $q_{e}^{i}$ induit par la circulation sur l'arc $e$ d'un container $(i=3)$ ou d'une remorque $(i=1)$ : ces derniers coûts sont des approximations, qui reflètent des surcoûts de consommation d'énergie et de qualité de service.

Pour tout couple $x, y$ dans $X$, on connaît la demande $D_{x, y}$ qui doit être acheminée de $x$ vers $y$ durant la période considérée. Cette demande est faite de containers, qui occupent le plus souvent $1 / 3$ de remorque), et de remorques entières, sachant qu'un camion ne peut tirer plus de 2 remorques.

Du fait de considérations sur la stabilité du système (aspect récurrent de la demande), on considère que la distribution des camions dans l'espace doit être stable au fil des périodes. Nous choisissons de modéliser ce point en assimilant la circulation des camions durant la période considérée à un flot entier $F \geq 0$, défini sur le réseau $G$.

L'acheminement de chaque demande $D_{x, y}$ est assimilée à un flot $f_{x, y} \geq 0$, fractionnaire, qui est un flot d'acheminement de la quantité $D_{x, y}$ de $x$ vers $y$. Ce flot doit pouvoir s'écrire comme une combinaison linéaire entière de 2 flots $f_{x, y}^{i}, i \in\{1,3\}$ dont les valeurs sont respectivement des multiples des nombres $1 / 2$, et $1 / 6$. L'ensemble des flots ainsi définis constitue un multiflot noté $f=\left(f_{x, y}^{i}, x, y \in X, i \in\{1,3\}\right)$.

Le flot $F$ doit dominer, sur chaque arc $e$, le flot agrégé $\operatorname{Sum}(f)=\Sigma_{x, y \in X} f_{x, y}$. $F$ et $f$ doivent être choisis de telle sorte que le coût $p . F+q . \operatorname{Sum}(f)$ soit le plus petit possible.

Pratiquement, sur une période considérée, les valeurs prises par $F$ ont vocation à être petites, ce qui rend mal adaptée l'approche qui consisterait à relaxer les contraintes d'intégrité sur $F$ et $f$.

Une formulation du problème est donc la suivante : (modèle LTL-MF :

Less than Truck Load Multicommodity flow)

$\left\{\right.$ Trouver, sur le réseau $G_{X}$, un flot $F$ entier $\geq 0$, et un multiflot

$f=\left(f_{x, y}^{i}, i \in\{1,3\}, x, y \in X\right) \geq 0$, tel que :

- pour tout couple $x, y \in X, f_{x, y}^{i}$ achemine la demande $D_{x, y}^{i}$ de $x$ vers $y$;

- pour tout $i \in\{1,3\}$, et tout $x, y \in X$, le flot 2i.f $x, y$ est entier ; (C4)

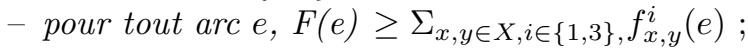

et qui minimise la quantité p.F + q.f.\}.

Il est important d'avoir conscience que ce modèle se contente de proposer des parcours pour les remorques et les containers, et de décrire la façon dont ces parcours peuvent être mutualisés. Il fait l'impasse sur les parcours effectifs des camions, et sur les aspects temporels, qui sont traités en aval du modèle LTL-MF de façon heuristique. 
Le problème ainsi formulé n'est pas exactement le problème CFEMF-OD, puisque le multiflot $f$ n'est pas simplement fractionnaire, mais doit satisfaire les contraintes $(\mathrm{C} 4)$, qui peuvent être considérées comme des contraintes d'intégrité. Toutefois, il devient exactement le problème CFEMF-OD dès lors que l'on fait l'hypothèse du chemin unique pour le multiflot $f$, et l'algorithme DME-CYGEN s'applique dès lors complètement.

Les tests réalisés concernent un réseau réel, prêté par le Département de Génie Industriel de l'Université d'Oklahoma, qui comporte 25 sites, 73 nœuds relais et $640 \operatorname{arcs}(\operatorname{Card}(X)=25+73=98, \operatorname{Card}(E)=640)$. Le nombre $K$ de couples origines-destination actifs, potentiellement égal à 1200, est ici égal à 392. Les jeux de demandes et de coûts sont quant à eux générés aléatoirement (chaque demande $D_{x, y}^{i}$ se situe entre 0 et 3 ), les demandes et coûts réels ne pouvant être prêtés. Les quantités calculées sont alors les suivantes :

- T-DME = temps CPU induit par l'exécution de l'algorithme DME-CYGEN;

- T-RE-LTL $=$ temps CPU induit par l'application de CPLEX au modèle LTL-MF, relaxé de la contrainte d'intégrité sur $F$ et $f$;

- GAP : écart, en pourcentage, entre les valeurs fournies par DME-CYGEN et par l'application de CPLEX à la relaxation fractionnaire du modèle LTL-MF.

Nous n'avons pas été en mesure d'obtenir la valeur optimale du modèle LTL-MF.

Trois résultats de tests sont présentés ici, numérotés de 1 à 3 . Les coûts $p_{e}$, $e \in E$, sont les mêmes pour chaque test, les coûts $q_{e}$ étant nuls pour le test 1 .

\begin{tabular}{|l|l|l|l|}
\hline Test & GAP & $T-D M E$ & $T-R E-L T L$ \\
\hline 1 & $10,6 \%$ & $42 \mathrm{~min}$ & $1 \mathrm{~h} 18 \mathrm{~min}$ \\
\hline 2 & $9,8 \%$ & $48 \mathrm{~min}$ & $1 \mathrm{~h} 15 \mathrm{~min}$ \\
\hline 3 & $8,9 \%$ & $46 \mathrm{~min}$ & $1 \mathrm{~h} 20 \mathrm{~min}$ \\
\hline
\end{tabular}

Commentaires : cette séquence de tests avait pour objectif principal de confronter les méthodes et modèles décrits dans les sections antérieures à des problèmes de tailles réelles, et d'évaluer notamment l'impact de ce facteur de taille sur les temps d'exécution. Dans le cas présent, on constate que cet impact est de fait assez important, les temps d'exécution associés aux tests ci-dessus étant trop élevés pour une intégration du modèle LTL-MF à l'intérieur d'un système de décision fonctionnant en temps continu. Par contre, on peut considérer que l'utilisation du modèle LTL-MF, à base de flots et de multiflots, et des méthodes que nous avons décrites ici, est viable dans la perspective d'une planification stratégique des activités du système de transport, effectuée sur une base de stationnarité de ses activités. Le traitement des routages et des charges effectives en temps réel (c'est-àdire, ici, avec des délais pour la prise de décision de l'ordre du quart d'heure), doit alors être envisagé de façon complémentaire à l'aide de processus moins lourds. Pour le reste, il ne nous était pas possible de comparer nos résultats avec ceux obtenus par les chercheurs de l'Université d'Oklahoma, car ceux-ci n'avaient pas inclus la contrainte de flot portant sur $F$ dans leur modèle. On remarquera toutefois que le gap entre les résultats fournis respectivement par la relaxation fractionnaire 
et par l'algorithme DME-CYGEN, s'est avéré ici sensiblement moindre que ceux enregistrés lors des tests de la section 5.4, et que ce gap tend à décroitre dès lors que l'on fait croître les coûts $q_{e}, e \in E$. Ceci tient au fait que les valeurs prises par le multiflot $f$ sont beaucoup plus grandes ici que dans les tests 5.4. On remarquera enfin que la méthode DME-CYGEN, qui est construite sur une hypothèse de routage mono-chemin, présente ici le grand avantage de prendre en compte de façon transparente les contraintes $(\mathrm{C} 4)$ qui pèsent ici sur le multiflot $f$.

\section{CONCLUSiON}

Nous avons présenté ici un formalisme général permettant de modéliser certains problèmes de couverture de flux par des infrastructures de transport à l'aide d'un flot «véhicule » entier et d'un multiflot « usager » fractionnaire, couplés par une contrainte de capacité. Nous avons d'abord proposé pour ces problèmes, divers schémas de décomposition et de relaxation, puis isolé un sous-problème de Flot de Coût Entier Minimum, et enfin introduit un mécanisme d'agrégation, qui nous ont permis de traiter le problème en considérant l'objet défini par le multiflot «usager $»$ comme objet principal, et en tirant parti de la structure particulière des espaces de flots.

Ce faisant, nous avons fait apparaitre différents problèmes auxiliaires, tels que celui de la recherche d'un circuit généralisé de coût négatif ou celui de la prise en compte, dans le cadre de la recherche d'un circuit négatif, de contraintes additionnelles associées à des coupes. Ces problèmes sont complexes et nous ne les avons traités que de façon heuristique. Il serait donc intéressant de les analyser de façon approfondie. De même il serait intéressant de reprendre le problème CFEMF en tenant compte d'élasticités des demandes d'acheminement de flux aux coûts individuels de parcours (les coûts $p(k), k \in K$ ), de façon à se rapprocher des réalités de certains problèmes de transport. Nous travaillerons enfin à l'avenir sur les possibilités d'applications des modèles et des techniques que nous venons d'introduire à des problèmes réels de transport et d'organisation de la production.

\section{RÉFÉRENCES}

[1] R.K. Ahuja, J.B. Orlin and D. Sharma, Multiexchance neighbourhood structures for the capacitated minimum spanning tree problem. Math Programming 91 (2001) 71-97.

[2] R.K. Ahuja, T.L. Magnanti, J.B. Orlin and M.R. Reddy, Applications of network optimization. Chapter 1 of Network Models, Handbook of Operation Research and Management Science 7 (1995)1-83.

[3] R.V. Ahuja, T.L. Magnanti and J.B. Orlin, Network Flows: Theory, Algorithms and Applications. Prentice hall, Englewood Cliffs, N.J. (1993).

[4] J.E. Aronson, A survey on dynamic network flows. Ann. Oper. Res. 20 (1989) 1-66.

[5] A. Assad, Multicommodity networks flows: a survey. Networks 8 (1978) 37-91.

[6] A. Balakrishnan, T. Magnanti and P. Mirchandani, Designing hierarchical survivable networks. Oper. Res. 46 (1998) 116-130.

[7] A. Balakrishnan, T. Magnanti and P. Mirchandani, A dual based algorithm for multi level network design. Manage. Sci. 40 (1994) 567-580. 
[8] M. Balinski, Fixed cost transportation problems. Nov. Res. Log. Quart 8 (1961) 41-54.

[9] F. Barahona, Network design using cut inequalities. SIAM J. Optim. 6 (1995) 822-837.

[10] W. Ben Ameur, Constrained length connectivity and survivable networks. Networks 36 (2000) 1.

[11] A. Benchakroun, J. Ferland and V. Gascon, Benders decomposition for network design problems with underlying tree structure. Investigacion operativa 6 (1997) 165-180.

[12] J.F. Benders, Partitionning procedures for solving mixed variable programming problems. Num. Math. 4 (1962) 238-252.

[13] D.Bertsimas and S. Stock Patterson, The air traffic flow problem with en route capacities. Oper. Res. 46-3 (1998) 406-422.

[14] D. Bienstock and O. Unluk, Capacited network design: polyedral structure and computation. Inform. J. Comput. 8 (1996) 243-259.

[15] T. Boffey and A. Hinxman, Solving for optimal network problem. EJOR 3 (1979) 386-393.

[16] A. Caminada, J.K. Hao, J.L. Lutton and V. Martin, L'optimisation des réseaux de télécommunications. Recherche Opérationnelle et Réseaux : Méthodes d'Analyse Spatiale. Collection IGAT, Hermes, Chap. 7 (2002) 191-236.

[17] S.G. Chang and B. Gavish, Telecommunication network topological design and capacity expansion: formulations and algorithms. Telecom. Syst. 1 (1993) 99-131.

[18] P. Chardaire, M.C. Costa and A. Sutter, Solving the dynamic facility location problem. Networks 28 (1996) 117-124.

[19] J. Chifflet, A. Lisser and P. Tolla, Interior point methods for multicommodity netflow problems. Perquisa Operacional 15 (1994) 1.

[20] S. Chopra and M. Rao, The Steiner tree problem I : formulations, composition and extension of facets. Math Programming 64 (1994) 209-229.

[21] N. Christophides, C.A. Whitlock, Network synthesis with connectivity constraint: a survey. Oper. Res. (1981) 705-723.

[22] J.P. Cordeau, P. Toth and D. Vigo, A survey of optimization models for train routing and scheduling. Transportation Science 32 (1998) 380-404.

[23] T. Crainic, M. Gendreau and J.M. Farvolden, A simplex based tabu search method for capacitated network design. Inform. J. Comput. 12 (2000) 223-236.

[24] T. Crainic and J.M. Rousseau, Multicommodity, multimode freight transportation: a general modeling and algorithmic framework for the service network design problem. Transport Research B 20 (1988) 290-297.

[25] T. Crainic, A. Frangioni and B. Gendron, Bundle based relaxation methods for multicommodity capacitated fixed charge network design. Discrete Appl. Math. 112 (2001) 73-99.

[26] G. Dahl and M. Stoer, A polyedral approach to multicommodity survivable network design. Numer. Math. 68 (1994) 149-167.

[27] P. Dejax and T. Crainic, A review of empty flow and fleet management models in freight transportation. Transportation Science 21 (1987) 227-247.

[28] D. De Wolf and Y. Smeers, Optimal dimensionning of pipe networks with application to gas transmission networks. Oper. Res. 44-4 (1996) 596-608.

[29] A. Dionne and M. Florian, Exact and approximate algorithms for optimal network design. Networks 9 (1979) 37-59.

[30] Z. Drezner and T. Drezner, Applied location theory models, in Modern Methods for Business Research, edited by Lawrence Erlbaum Mahwa, N.J. (1998) 79-120.

[31] H.A. Eiselt, G. Laporte and J.F. Thisse, Competitive location models: a framework and bibliography. Transportation Sciences 27 (1993) 44-54.

[32] V. Ferreira Filho and J. Galvao, A survey of computer network design problems. Investigacion Operativa 4 (1994) 183-211.

[33] M. Florian, An introduction to network models used in transportation planning, in Transp. Plann. Models, edited by M. Florian, North Holland, Amsterdam (1984) 137-152.

[34] J.M. Forvalden, W.B. Powell and I. Lustig, A primal partitionning solution for the arc chain formulation of a multicommodity network flow problem. Oper. Res. 41 (1993) 669-693.

[35] G. Gallo, Lower planes for the network design problem. Networks 13 (1983) 411-426. 
[36] B. Gavish, Topological design of telecommunication networks: local access design methods. Ann. Oper. Res. 33 (1991) 17-71.

[37] A. Geoffrion, Lagrangean relaxation and its uses in integer programming. Math. Prog. Study 2 (1974) 82-114.

[38] A. Geoffrion, Generalized Benders decomposition. J. Optim. Theory Appl. 10 (1972) $237-260$.

[39] A. Girard and B. Liau, Dimensioning of adaptatively routed networks. IEE/ACM Trans. Networking 1-4 (1993) 460-468.

[40] A. Golberg and R. Tarjan, Finding minimum cost circulation by cancelling negative cycles, JACM 36 (1989) 873-886.

[41] H. Hoang, Topological optimization of networks: a non linear mixed model employing generalized Benders decomposition. IEEE Trans. Automat. Control. AC-27 (1982) 164-169.

[42] P.A. Hossein, D.P. Bertsekas and P. Tseng, Relaxation methods for network problems with convex arc costs. SIAM J. Control Optim. 5 (1987) 1219-1243.

[43] F.K. Hwang, D.S. Richards and P. Winter, The Steiner Tree Problem. North Holland (1992).

[44] P. Jaillet, G. Song and G. Yu, Airline network design and hub location problems. Location Science 4 (1997) 195-212.

[45] D. Johnson, J. Lenstra, A. RInnoy-Khan, The complexity of the Network Design Problem. Networks 8 (1978) 279-285.

[46] K.L. Jones, I.J. Lustig, J.M. Farvolden and W.B. Powel, Multicommodity network flows: the impact of formulation on decomposition. Math. Programming 62 (1993) 95-117.

[47] J.L. Kennington and R.V. Helgason, Algorithms for network programming. Wiley N.Y. (1980).

[48] B.M. Khumalala, Warehouse location problem efficient branch and bound algorithm. $M a-$ nage. Sciences B $\mathbf{1 8}$ (1972) 718-731.

[49] J.G. Klincewicz and M.B. Rosenwein, Planning and consolidating shipments from a warehouse. J. Operat. Res. Soc. 48 (1997) 241-246.

[50] L.J. Leblanc, An algorithm for discrete network design. Trans. Sci. 9 (1975) 283-287.

[51] P.J. Lederer and R.S. Nambimadom, Airline network design. Oper. Res. 46-6 (1998) 785804.

[52] J. Mac Gregor Smith and P. Winter, Topological Network Design. Ann. Oper. Res. 33 (1991).

[53] T. Magnanti, Combinatorial optimization and vehicle fleet planning: perspectives and prospects. Networks 11 (1981) 179-214.

[54] T. Magnanti and R.T. Wong, Network design and transportation planning models and algorithms. Trans. Sci. 18 (1984) 1-5.

[55] P. Mahey, A. Benchakroun and F. Boyer, Capacity and flow assignment of data networks by generalized Benders decomposition. J. Global Optim. 20 (2001) 173-193.

[56] A. Marin and J. Salmeron, Tactical design of rail freight networks: part 1- exact and heuristic methods. EJOR 90 (1996) 26-44.

[57] M. Minoux, Network synthesis and optimum network design problems: models, solution methods and application. Networks 19 (1989) 313-360.

[58] M. Minoux, Optimum synthesis of a network with non simultaneous multicommodity flow requirements, Studies on graphs and Discrete Programming, Annals of Disc. Math., edited by P. Hansen, North Holland 11 (1981) 269-277.

[59] P.B. Mirchandani and L.R. Francis, Discrete Location Theory. John Wiley Eds, N.Y. (1990).

[60] I. Norenkov, Y. Yevstifeyev and V. Manichev, A method for an accelerated analysis of multiperiod electronic circuits. Telecom Radio Engin. 42 (1987) 123-126.

[61] A. Ouorou, P. Mahey and J.P. Vial, A survey of algorithms for convex multicommodity flow problems. Manage. Science 46 (2000) 126-147.

[62] P.M. Pardalos and D.Z. Du, Network design: connectivity and facility location. DIMACS Series 40, N.Y., American Math Society (1998).

[63] R. Rebai, Optimisation de réseaux de télécommunications avec sécurisation. Thèse ParisDauphine (2000). 
[64] T. Scott and E. Read, Modelling hydroreservoir operation in a deregulated electricity market. ITOR 3 (1996) 243-253.

[65] P.A. Steenbrink, Optimization of transport networks. Wiley, N.Y. (1974).

[66] P. Tseng, Dual Ascent methods for problems with strictly convex costs and linear constraints: a unified approach. SIAM J. Control Optim. 28 (1990) 214-242.

[67] R. Vijay, A. Kanda and P. Vrat, Multiperiod capacity expansion of road networks: formulation and algorithms. Oper. Res. 30 (1993) 117-140.

[68] R.T. Wong, Worst case analysis of network design problem heuristics. SIAM J. Alg. Disc. Meth. 1 (1980) 51-63. 